Department of Policy and Planning Sciences

\author{
Discussion Paper Series
}

No.1333

Pure-strategy Nash equilibria on competitive diffusion games

by

Hikoe ENOMOTO, Masahiro HACHIMORI, Shun NAKAMURA, Maiko SHIGENO, Yuya TANAKA, and Masakl TSUGAMI

Jul 2015

UNIVERSITY OF TSUKUBA

Tsukuba, Ibaraki 305-8573

JAPAN 


\title{
Pure-strategy Nash equilibria on competitive diffusion games
}

\author{
Hikoe Enomoto, Masahiro Hachimori, Shun Nakamura, Maiko Shigeno, \\ Yuya TANakA and Masaki Tsugami
}

June 20, 2015

\section{Introduction}

Competitive facility location games are the models of rivaling sellers seeking positions to maximize their market share. A foundation of competitive facility location games is known as the ice-cream vender problem by Hotelling [5]. Recently, competitive facility location games on graphs are widely studied $[2,4,11]$. Such a game on a graph can model, for example, the process that rumors about some products spread through social networks. Each competitive firm wants to select several influencers from whom information diffuse throughout the network efficiently.

In this paper, we focus on two kinds of competitive facility location games. One is the information diffusion game, which is introduced by Alon et al. [1], and the other is the discrete Voronoi game discussed by Dürr and Tang [3]. In both of these games, each player selects one vertex on a given graph, and sends his/her message to this selected vertex. After that, each informed vertex sends the received message to all its uninformed neighbors in each step. The utility of each player is given by the number of vertices received his/her message when all the diffusion process is finished. The difference of the two models is the behavior of the vertices which receive more than one message at the same time. In the former model, messages vanish due to collisions of messages, and the diffusion process is prevented at the vertices where collisions occur. In the latter model, on the other hand, when more than one message arrives to one vertex at the same time, the vertex receives all of them and the utility is shared equally. Thus, all vertices contribute to the utility of some players. Note that the game proposed by [10] is different from our discrete Voronoi game although it uses the same name.

We investigate the existence of Nash equilibria for our games. Nash equilibria are the stable states of the game in which no player can improve his/her utility by unilaterally changing to a different strategy. Determining whether Nash equilibria exist and effectively computing them have attracted many researchers in economics, decision making and computer science. Our interest is on the existence of Nash equilibria in the case of pure strategies, where each player chooses an action deterministically. We do not deal with mixed strategies in this paper. Nash equilibria for pure strategies are briefly referred to as pure Nash equilibria. The existences of pure Nash equilibria for information diffusion games and discrete Voronoi games are studied by [1,3]. Dürr and Thang [3] showed a relatively simple graph that does not allow a pure Nash equilibrium for Voronoi games even though there are only two players. 
This paper characterizes the existence of pure Nash equilibria on small diameter graphs, path graphs and cycle graphs. After giving formal definitions of information diffusion games and discrete Voronoi games in Section 2, we discuss the existence of the pure Nash equilibria for graphs with small diameters in Section 3. In Section 4, we show the complete characterization of the existence of the pure Nash equilibria in the discrete Voronoi games and information diffusion games on path graphs. We close the discussion in Section 5 with some observations for the discrete Voronoi games on trees.

\section{Information diffusion game and discrete Voronoi game}

Let $G=(V, E)$ be an undirected graph and $N$ a set of players. Throughout this paper we always assume $G$ is connected. Suppose that the graph $G$ has $n$ vertices and there are $k$ players, that is, $|V|=n$ and $|N|=k$. In both games, a strategy set of each player is $V$ and a strategy profile of players is a vector $\boldsymbol{x} \in V^{N}$ associating each player to a vertex selected initially.

In the information diffusion game induced by $G$, each player having own message wants to spread his/her message to as many vertices in graph $G$ as possible. Each vertex admits three states: uninformed, informed, and deadlocked. Initially, every vertex is uninformed. At time one, each player selects one vertex from $V$ and sends his/her message to the selected vertex. Then, a vertex that is sent only one message, i.e., a vertex selected by exactly one player, receives the message and turns into the state of informed, while a vertex that is sent more than one message becomes deadlocked. At time $t+1$, each informed vertex that received a message at time $t$ sends the same message to all of its adjacent uninformed vertices, and these messages are processed in the same way: An uninformed vertex that is sent only one message receives the message and turns into the state of informed, while an uninformed vertex that is sent more than one message becomes deadlocked. Deadlocked vertices do not receive any messages. This diffusion process finishes when there is no vertex that newly becomes informed. Given a strategy profile $\boldsymbol{x}$ which stands for a vertex selected by each player at time one, the utility of player $i$, denoted by $U_{i}(\boldsymbol{x})$, is given by the number of informed vertices that receive the message of player $i$ at the end of the diffusion process.

In the discrete Voronoi game, we use a notation $C_{v}(\boldsymbol{x})$, for a vertex $v$ and a strategy profile $\boldsymbol{x}=\left(x_{1}, \ldots, x_{k}\right)$, to represent the set of players $i$ whose selected vertex $x_{i}$ is closest to $v$ among $x_{1}, \ldots, x_{k}$. Namely, $C_{v}(\boldsymbol{x})=\arg \min \left\{d\left(x_{i}, v\right) \mid i \in N\right\}$, where $d(u, v)$ denotes the shortest path length between $u \in V$ and $v \in V$ in $G$. The utility $\tilde{U}_{i}(\boldsymbol{x})$ of player $i$ in the discrete Voronoi game is given by $\sum\left\{\frac{1}{\left|C_{v}(\boldsymbol{x})\right|} \mid v \in V, i \in C_{v}(\boldsymbol{x})\right\}$.

The information diffusion game and the discrete Voronoi game are similar in the sense that every vertex is assigned to the closest players and utility is given by the number of vertices assigned to each player. The difference of these two games happens when a vertex receives more than one message simultaneously. Such vertices will be shared by the players and the messages will be propagated further in the discrete Voronoi game, while they remain just in the deadlocked state and the propagation of the messages stops at them in the information diffusion game. Especially, in the discrete Voronoi game we always have $\sum_{i \in N} \tilde{U}_{i}(\boldsymbol{x})=|V|$ for any $\boldsymbol{x}$, which does not necessarily hold in the information diffusion game. 
Given a strategy profile $\boldsymbol{x}=\left(x_{1}, \ldots, x_{k}\right)$ and $x^{\prime} \in V$, we denote by $\left(x^{\prime}, \boldsymbol{x}_{-i}\right)$ the vector equal to $\boldsymbol{x}$ but with the $i$ th component replaced by $x^{\prime}$, that is, $\left(x^{\prime}, \boldsymbol{x}_{-i}\right)=\left(x_{1}, \ldots, x_{i-1}, x^{\prime}\right.$, $\left.x_{i+1}, \ldots, x_{k}\right)$. A strategy profile $\boldsymbol{x}$ is a pure Nash equilibrium of the information diffusion game (resp. the discrete Voronoi game), if $U_{i}\left(x^{\prime}, \boldsymbol{x}_{-i}\right) \leq U_{i}(\boldsymbol{x})\left(\operatorname{resp} . \tilde{U}_{i}\left(x^{\prime}, \boldsymbol{x}_{-i}\right) \leq \tilde{U}_{i}(\boldsymbol{x})\right)$ for any player $i$ and any $x^{\prime} \in V$.

Throughout this paper, we assume that $k \leq n$, otherwise both games have trivial structures. Namely, a profile is a Nash equilibrium of the information diffusion game if and only if there are no unoccupied vertices, and it is a Nash equilibrium of the discrete Voronoi game if and only if the numbers of players located on the vertices differ by at most one between any two vertices.

\section{Existence of pure Nash equilibria on graphs with small diameter}

This section discusses the existence of pure Nash equilibria of our games induced by graphs having small diameter, which was firstly discussed by Alon et al.[1] for information diffusion games. The diameter of a graph is defined by the maximum distance between a pair of vertices, that is, defined by $\max \{d(v, u) \mid u, v \in V\}$. Alon et al. have shown that the information diffusion game on a graph whose diameter is at least three may not possess a pure Nash equilibrium, even for two players. Although their example is given by a large graph, the smaller graph shown in Figure 1 is also an example on which the information diffusion game does not admit a pure Nash equilibrium. Moreover, Takehara et al. [9] have shown the following.

Theorem 1 ([9]) There exists a graph with diameter two such that the information diffusion game induced by it does not admit a pure Nash equilibrium even for two players.

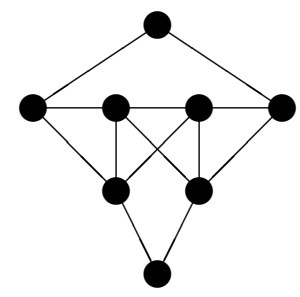

Figure 1: An Example where Nash equilibria of the information diffusion game does not exist for two players.

Note that the utilities of the information diffusion games are not given by only distances of pairs of vertices. Hence, characterization of equilibria by some indicators of graph distances seems to be difficult. On the other hand, in the discrete Voronoi games, the utilities are defined by distances of pairs of vertices. Indeed, the diameters are able to characterize the existence of pure Nash equilibrium for discrete Voronoi games.

Theorem 2 For two players, the discrete Voronoi game induced by any graph with diameter two admits a pure Nash equilibrium. 
Proof. Let $\tilde{v}$ be a vertex of the maximum degree. We shall show that the strategy profile $(\tilde{v}, \tilde{v})$ is a pure Nash equilibrium.

Assume that there exists a vertex $x$ with $\tilde{U}_{1}(x, \tilde{v})>\tilde{U}_{1}(\tilde{v}, \tilde{v})$. Let $\Gamma(v)$ be the closed neighbor of a vertex $v \in V$, that is, $\Gamma(v)=\{u \in V \mid(u, v) \in E\} \cup\{v\}$. Note that $d(x, v)=d(\tilde{v}, v)=2$ holds for any $v \notin \Gamma(x) \cup \Gamma(\tilde{v})$, since the diameter is two. Thus, we have

$$
\tilde{U}_{1}(x, \tilde{v})-\tilde{U}_{2}(x, \tilde{v})=|\Gamma(x) \backslash \Gamma(\tilde{v})|-|\Gamma(\tilde{v}) \backslash \Gamma(x)|,
$$

which, together with $\tilde{U}_{1}(x, \tilde{v})>\tilde{U}_{1}(\tilde{v}, \tilde{v})=\tilde{U}_{2}(\tilde{v}, \tilde{v})>\tilde{U}_{2}(x, \tilde{v})=|V|-\tilde{U}_{1}(x, \tilde{v})$, implies that $|\Gamma(x) \backslash \Gamma(\tilde{v})|>|\Gamma(\tilde{v}) \backslash \Gamma(x)|$. Thus, we obtain $|\Gamma(x)|>|\Gamma(\tilde{v})|$, which contradicts the maximality of the degree of $\tilde{v}$.

For three players, there exists a graph with diameter two such that the discrete Voronoi game induced by it does not admit a pure Nash equilibrium. For example, the cycle graph with 4 vertices does not admit a pure Nash equilibrium for three players, as shown in [7]. More generally, we have the following.

Theorem 3 For any $k \geq 3$, there exist graphs of diameter 2 on which the discrete Voronoi games do not admit pure Nash equilibria for $k$ players.

Proof. Consider the join $C_{t}+\bar{K}_{s}$ of the cycle $C_{t}$ of size $t$ and the complement $\bar{K}_{s}$ of the complete graph of size $s$, i.e., the graph obtained from the cycle $C_{t}$ by adding vertices $v_{1}, v_{2}, \ldots, v_{s}$ and edges $\left(v, v_{i}\right)$ for each vertex $v$ in $C_{t}$ and $v_{i}$ for $i=1, \ldots, s$ (see Figure 2). Note that the diameter of $C_{t}+\bar{K}_{s}$ is two for any $t(\geq 4)$ and $s(\geq 1)$. We show that this graph with $t=k+1$ and $s$ large enough, i.e., the graph $C_{k+1}+\bar{K}_{M}$ with $M \geq 2 k+1$, does not admit any pure Nash equilibria for $k$ players.

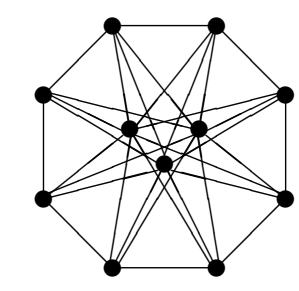

Figure 2: Example for $C_{t}+\bar{K}_{s}$, where $t=8$ and $s=3$.

Consider a strategy profile such that there are $p$ players on the cycle $C_{k+1}$ and $k-p$ players on $\bar{K}_{M}$, where $0 \leq p \leq k$. Assume this strategy profile is a pure Nash equilibrium.

When $p=0$, for the utility of a player $i$ on $\bar{K}_{M}$, the vertex where $i$ locates is shared by the players on the vertex, and the $k+1$ vertices on $C_{k+1}$ and the unoccupied vertices on $K_{M}$ are also shared by $k$ players. Thus the utility of the player $i$ equals to $\frac{1}{h}+\frac{k+1}{k}+\frac{\ell}{k}$, where $h$ is the number of players on the vertex where $i$ locates and $\ell$ is the number of unoccupied vertices on $\bar{K}_{M}$. We have $h \leq k$ and $\ell \geq 1$. Here, if $h \geq 2$ and the player $i$ relocates to an unoccupied vertex on $\bar{K}_{M}$, then his/her utility becomes $1+\frac{k+1}{k}+\frac{\ell-1}{k}$ which is larger than the original utility $\frac{1}{h}+\frac{k+1}{k}+\frac{\ell}{k}$. This contradicts the assumption that the profile is a pure Nash equilibrium. Hence we can assume that all the players locate on different vertices on $\bar{K}_{M}$. In such a strategy profile, the utility $\tilde{U}_{i}$ of a player $i$ equals to 
$\frac{(k+1)+M}{k}$. However, if this player $i$ relocates to a vertex on $C_{k+1}$, his/her utility becomes $\tilde{U}_{i}^{\prime}=1+\frac{2}{k}+(M-(k-1))>M-k+2$. Hence we have

$$
\tilde{U}_{i}^{\prime}-\tilde{U}_{i}>(M-k+2)-\frac{(k+1)+M}{k}=\frac{(k-1) M-k^{2}+k-1}{k},
$$

which is positive when $M \geq\left\lceil k+\frac{1}{k-1}\right\rceil=k+1$. Since we have $M \geq 2 k+1 \geq k+1$, this contradicts the assumption that the profile is a pure Nash equilibrium.

When $1 \leq p \leq k-1$, we observe that each of the $p$ players on $C_{k+1}$ receives at least 1 from the vertices of $C_{k+1}$ as his/her utility, since otherwise he/she can increase his/her utility by relocating to an unoccupied vertex on $C_{k+1}$. This implies that the $k-p$ players on $\bar{K}_{M}$ shares at most $(k+1)-p$ from the vertices of $C_{k+1}$ as their utility. Hence there exists a player $j$ whose utility $\tilde{U}_{j}$ is at most $1+\frac{(k+1)-p}{k-p}$. However, if the player $j$ relocates to an unoccupied vertex of $C_{k+1}$, his/her utility $\tilde{U}_{j}^{\prime}$ becomes at least $1+\frac{M-(k-(p+1))}{p+1}$. Hence we have

$$
\begin{aligned}
\tilde{U}_{j}^{\prime}-\tilde{U}_{j} & \geq\left(1+\frac{M-(k-(p+1))}{p+1}\right)-\left(1+\frac{(k+1)-p}{k-p}\right) \\
& =\frac{M-k}{p+1}-\frac{1}{k-p} \geq \frac{M-k}{(k-1)+1}-\frac{1}{k-(k-1)}=\frac{M-2 k}{k},
\end{aligned}
$$

which is positive for $M \geq 2 k+1$, a contradiction.

When $p=k$, every player locates on the cycle $C_{k+1}$. Here, we observe the following (i) - (iii). Note that all the players on the cycle equally share the vertices on $\bar{K}_{M}$ as their utilities.

(i) Vertices chosen by no players do not appear sequentially.

Assume two vertices $v$ and $v^{\prime}$ that are not chosen by any players appear sequentially on the cycle. If one player relocates to $v$, then,as his/her utility, he/she gets 1 from $u$ and at least $1 / k$ from $v^{\prime}$. The case the utility from $v^{\prime}$ equals to $1 / k$ occurs only when all the other $k-1$ players on the cycle locates on the vertex next to $v^{\prime}$. In such a case, $v$ has one more unoccupied vertex than $v^{\prime}$ in its neighbor and he/she gets additionally as his/her utility. Hence his/her utility received from the vertices of the cycle is strictly larger than $1+1 / k$. On the other hand, the $k$ players share $k+1$ vertices on the cycle. This implies there exists a player whose utility from the cycle is at most $(k+1) / k=1+1 / k$. If such a player moves to $v$, then his/her utility increases, contradicting the assumption that the profile is a pure Nash equilibrium.

(ii) No vertices are chosen by more than two players.

Assume there is a vertex $v$ on the cycle on which three or more players locates. Then, for his/her utility from the cycle, he/she gets at most $1 / 3$ from $v$, and at most $1 / 4$ (by (i)) from each of its neighbors if they are unoccupied. So in total he/she gets at most $5 / 6<1$ from the cycle. If he/she relocates to an unoccupied vertex on the cycle, his/her utility from the cycle becomes at least 1 and his/her utility increases, contradicting to the assumption that the profile is a pure Nash equilibrium.

(iii) Both sides of a vertex located by two players are not chosen by any player.

Assume two players locate on a vertex $v$ on the cycle. Then a player on $v$ receives, 
as his/her utility, $1 / 2$ from $v$, and at most $1 / 3$ (by (i)) from its neighbor vertex $v^{\prime}$ on the cycle if $v^{\prime}$ is unoccupied. Hence, if only one neighbor vertex is unoccupied then the utility from the cycle is less than 1 . If so, he/she can increase his/her utility by moving to an unoccupied vertex on the cycle, contradicting to the assumption that the profile is a pure Nash equilibrium.

For a pair of adjacent vertices on $C_{k+1}$, consider the sum of the numbers of players located on the two vertices. There are $k+1$ such pairs and the total sum of the values equals to $2 k$, thus the avarage is $\frac{2 k}{k+1}<2$. Hence, together with (i), we conclude there exists a pair of adjacent vertices on $C_{k+1}$ such that the sum of the numbers of players located on them is 1 . Assume $v_{1}$ and $v_{2}$ are such adjacent vertices that exactly one player is on $v_{1}$ and no player is on $v_{2}$. Let $v_{3}$ be the vertex on $C_{k+1}$ next to $v_{2}$. By (i) and (ii), the number of players on $v_{3}$ is either one or two. If there is exactly one player on $v_{3}$, then each of the players on $v_{1}$ and $v_{3}$ takes at least $\frac{3}{2}$ as his/her utility from $C_{k+1}$. Since the $k+1$ vertices of $C_{k+1}$ are shared by $k$ players and every player takes at least 1 as his/her utility from $C_{k+1}$, each of the players on $v_{1}$ and $v_{3}$ takes exactly $\frac{3}{2}$ and the others takes exactly 1 for each as their utility from $C_{k+1}$. This can occur only when on each vertices other than $v_{2}$ exactly one player locates. But, if so, the player on $v_{4}$ next to $v_{3}$ can increase his/her utility by relocating to $v_{3}$, a contradiction. Assume there are two players on $v_{3}$. Let $v_{0}$ be the vertex on $C_{k+1}$ previous to $v_{1}$, and $v_{-1}$ be the one previous to $v_{0}$. By (i) and (iii), there are two cases: exactly one player is on $v_{0}$, or no player is on $v_{0}$ and some players are on $v_{-1}$. In both cases, the player on $v_{1}$ can increase his/her utility by relocating to $v_{2}$, a contradiction.

Moreover, there is a graph with diameter three such that the discrete Voronoi game induced by it does not admit a pure Nash equilibrium for two players. The graph given by Figure 4 in [3] is such an example.

\section{Existence of pure Nash equilibria on path graphs}

This section deals with the games induced by a path graph $G=(V, E)$. For convenience, assume that $V$ is given by a range of integers from 1 to $n$, that is, $V=\{1,2, \ldots, n\}$ and $E=\{(i, i+1) \mid i=1, \ldots, n-1\}$. We assume, as is remarked in the end of Section 2, the number $k$ of the players of the games is at most $n$.

One may expect that, on path graphs, the discrete Voronoi game and the information diffusion game have similar equilibrium structures since it seems that the difference is just that the deadlocked vertices in the diffusion process game are shared by the closest players in the discrete Voronoi game. However, contrary to this expectation, the structures of pure Nash equilibria of these two games can be quite different. For example, let us consider the games on the path with nine vertices and five players (see Figure 3). The strategy profile $\boldsymbol{x}=(2,3,5,7,8)$ is a pure Nash equilibrium for the discrete Voronoi game. The utility of each player is $\tilde{U}_{1}(\boldsymbol{x})=\tilde{U}_{3}(\boldsymbol{x})=\tilde{U}_{5}(\boldsymbol{x})=2$ and $\tilde{U}_{2}(\boldsymbol{x})=\tilde{U}_{4}(\boldsymbol{x})=1.5$. However, this strategy profile $\boldsymbol{x}$ is not a pure Nash equilibrium for the information diffusion game, since $\boldsymbol{x}^{\prime}=(2,3,4,7,8)$ has the utility $U_{3}\left(\boldsymbol{x}^{\prime}\right)=2$ which is greater than $U_{3}(\boldsymbol{x})=1$. On the other hand, although $\boldsymbol{x}^{\prime}$ is a pure Nash equilibrium for the information diffusion game, it is not a pure Nash equilibrium for the discrete Voronoi game, since $\boldsymbol{x}^{\prime \prime}=(2,5,4,7,8)$ has the utility $\tilde{U}_{2}\left(\boldsymbol{x}^{\prime \prime}\right)=1.5$ which is greater than $\tilde{U}_{2}\left(\boldsymbol{x}^{\prime}\right)=1$. It is worth investigating the difference 


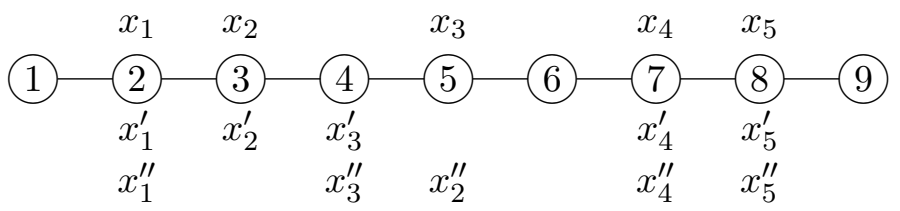

Figure 3: An example where Nash equilibria of the information diffusion game and the Voronoi game are different.

of pure Nash equilibria between the discrete Voronoi games and the information diffusion games on the path graphs in detail. In the following, we determine when the games on path graphs have pure Nash equilibria: for the discrete Voronoi game in Section 4.1, and for the information diffusion game in Section 4.2.

As used in $[3,7]$, let $\theta_{1}, \ldots, \theta_{\ell}$ be the distinct vertices chosen by some players in the strategy profile under consideration. Assume that $\theta_{1}<\theta_{2}<\cdots<\theta_{\ell}$. For each $j=1, \ldots, \ell$, let $c_{j}$ be the number of players who choose the vertex $\theta_{j}$. Note that a pair of $\boldsymbol{\theta}=\left(\theta_{1}, \ldots, \theta_{\ell}\right)$ and $\boldsymbol{c}=\left(c_{1}, \ldots, c_{\ell}\right)$ represents the strategy profile completely, where $\sum_{j} c_{j}$ equals to the number $k$ of players. We denote the set of vertices between $\theta_{j}$ and $\theta_{j+1}$ by $L_{j}$. That is,

$$
L_{j}= \begin{cases}\left\{i \in \mathbb{Z} \mid 1 \leq i<\theta_{1}\right\} & (j=0) \\ \left\{i \in \mathbb{Z} \mid \theta_{j}<i<\theta_{j+1}\right\} & (1 \leq j \leq \ell-1) \\ \left\{i \in \mathbb{Z} \mid \theta_{j}<i \leq n\right\} & (j=\ell) .\end{cases}
$$

Note that $\left(L_{0}, \ldots, L_{\ell}\right)$ has one-to-one correspondence to $\boldsymbol{\theta}$.

For any integer $m$, define

$$
\delta(m)= \begin{cases}0 & (m: \text { even }) \\ 1 & (m: \text { odd }) .\end{cases}
$$

\subsection{Discrete Voronoi game}

With respect to a strategy profile $(\boldsymbol{\theta}, \boldsymbol{c})$ for the discrete Voronoi game, the utility of a player who chooses the vertex $\theta_{j}$ is given by

$$
\tilde{u}_{j}(\boldsymbol{\theta}, \boldsymbol{c})= \begin{cases}\frac{1}{c_{1}}\left(\left|L_{0}\right|+1+\left\lfloor\frac{\left|L_{1}\right|}{2}\right\rfloor\right)+\frac{\delta\left(\left|L_{1}\right|\right)}{c_{1}+c_{2}} & (j=1) \\ \frac{\delta\left(\left|L_{j-1}\right|\right)}{c_{j-1}+c_{j}}+\frac{1}{c_{j}}\left(\left\lfloor\frac{\left|L_{j-1}\right|}{2}\right\rfloor+1+\left\lfloor\frac{\left|L_{j}\right|}{2}\right\rfloor\right)+\frac{\delta\left(\left|L_{j}\right|\right)}{c_{j}+c_{j+1}} & (1<j<\ell) \\ \frac{\delta\left(\left|L_{\ell-1}\right|\right)}{c_{\ell-1}+c_{\ell}}+\frac{1}{c_{\ell}}\left(\left\lfloor\frac{\left|L_{\ell-1}\right|}{2}\right\rfloor+1+\left|L_{\ell}\right|\right) & (j=\ell)\end{cases}
$$

provided that $\ell \geq 2$. For short, if there is no confusion, we write $\tilde{u}_{j}$, instead of $\tilde{u}_{j}(\boldsymbol{\theta}, \boldsymbol{c})$.

We first give a characterization of pure Nash equilibria for the discrete Voronoi games on path graphs. This characterization is obtained from a modification of the property for the discrete Voronoi games on cycle graphs by $[3,7]$.

Theorem 4 For the discrete Voronoi game with $k$ players on a path graph with $n(\geq$ 4 , and, $\geq k)$ vertices, a strategy profile defined by $(\boldsymbol{\theta}, \boldsymbol{c})$ is a pure Nash equilibrium if and only if the following holds: 
(V1) $c_{j} \leq 2$ for all $j=1, \ldots, \ell$.

(V2) $\left|L_{j}\right| \leq\lfloor 2 \tilde{\gamma}\rfloor-1$ for all $j=1, \ldots, \ell-1,\left|L_{0}\right| \leq\lfloor\tilde{\gamma}\rfloor$ and $\left|L_{\ell}\right| \leq\lfloor\tilde{\gamma}\rfloor$.

(V3) If $c_{j} \neq c_{j+1}$, then $\left|L_{j}\right|$ is even, for all $j=1, \ldots, \ell-1$, except in two cases: when $c_{1} \geq 2, c_{2}=1$ and $c_{3} \geq 2$, and when $c_{\ell} \geq 2, c_{\ell-1}=1$ and $c_{\ell-2} \geq 2$. In the former case, at least one of $\left|L_{1}\right|$ and $\left|L_{2}\right|$ is even. Symmetrically, in the latter case, at least one of $\left|L_{\ell-2}\right|$ and $\left|L_{\ell-1}\right|$ is even.

(V4) If $c_{j}=1$ and $\left|L_{j-1}\right|=\left|L_{j}\right|=2 \tilde{\gamma}-1$ for some $j=2, \ldots, \ell-1$, then $2 \tilde{\gamma}$ is odd.

(V5) (i) If $c_{j}=c_{j+1}=1$ and $\left|L_{j-1}\right|+\left|L_{j}\right|+1=\left|L_{j+1}\right|=2 \tilde{\gamma}-1$ for some $j=2, \ldots, \ell-2$, then $2 \tilde{\gamma}$ is odd.

(ii) If $c_{j-1}=c_{j}=1$ and $\left|L_{j-1}\right|=\left|L_{j}\right|+\left|L_{j+1}\right|+1=2 \tilde{\gamma}-1$ for some $j=2, \ldots, \ell-2$, then $2 \tilde{\gamma}$ is odd.

(V6) If $c_{1}=1$ (resp. $\left.c_{\ell}=1\right)$, then $\left|L_{1}\right|=0$ (resp. $\left|L_{\ell-1}\right|=0$ ). Additionally, $\left|L_{0}\right|=$ $\left\lfloor\frac{\left|L_{2}\right|+1}{2}\right\rfloor$ (resp. $\left|L_{\ell}\right|=\left\lfloor\frac{\left|L_{\ell-2}\right|+1}{2}\right\rfloor$ ) provided that $\ell \geq 3$.

Here, $\tilde{\gamma}=\min \left\{\tilde{u}_{j} \mid j=1, \ldots, \ell\right\}$ is the minimum utility.

The proof of this theorem is given in Appendix. (The statement of this theorem holds for $2 n \geq k$, but it is stated for $n \geq k$ here since we are assuming $n \geq k$ throughout this paper. In the appendix, the proof is given for $2 n \geq k$.)

Lemma 5 (i) If (V2) is fulfilled, then $\left|L_{j-1}\right|=\left|L_{j}\right|=\lfloor 2 \tilde{\gamma}\rfloor-1$ holds when $c_{j} \geq 2$ for $j=2, \ldots, \ell-1$.

(ii) If (V2) is fulfilled and $\ell \geq 2$, then $\left|L_{0}\right|=\lfloor\tilde{\gamma}\rfloor$ and $\left|L_{1}\right| \geq\lfloor 2 \tilde{\gamma}\rfloor-2$ hold when $c_{1} \geq 2$. Symmetrically, we have $\left|L_{\ell}\right|=\lfloor\tilde{\gamma}\rfloor$ and $\left|L_{\ell-1}\right| \geq\lfloor 2 \tilde{\gamma}\rfloor-2$ hold when $c_{\ell}>2$.

(iii) If (V2) and (V6) are fulfilled and $\ell \geq 3$, then $c_{2}=1$ when $c_{1}=1$. Symmetrically, we have $c_{\ell-1}=1$ when $c_{\ell}=1$.

Proof. (i) Consider a strategy profile satisfying (V2). Suppose that there exists $j$ such that $c_{j} \geq 2$ and $\left|L_{j}\right| \leq\lfloor 2 \tilde{\gamma}\rfloor-2$. Then, we have

$$
\tilde{u}_{j} \leq \frac{\delta(\lfloor 2 \tilde{\gamma}\rfloor-1)}{3}+\frac{\left\lfloor\frac{\lfloor 2 \tilde{\gamma}\rfloor-1}{2}\right\rfloor+1+\left\lfloor\frac{\lfloor 2 \tilde{\gamma}\rfloor-2}{2}\right\rfloor}{2}+\frac{\delta(\lfloor 2 \tilde{\gamma}\rfloor-2)}{3} .
$$

Since either $\lfloor 2 \tilde{\gamma}\rfloor-1$ or $\lfloor 2 \tilde{\gamma}\rfloor-2$ is odd, we obtain

$$
\tilde{u}_{j} \leq \frac{1}{3}+\tilde{\gamma}-\frac{1}{2}<\tilde{\gamma}
$$

which contradicts the minimality of $\tilde{\gamma}$.

(ii) Consider a strategy profile satisfying (V2). Suppose that $c_{1} \geq 2$. If $\left|L_{0}\right| \leq\lfloor\tilde{\gamma}\rfloor-1$, then we have

$$
\begin{aligned}
\tilde{u}_{1} & \leq \frac{1}{2}\left(\left|L_{0}\right|+1+\left\lfloor\frac{\left|L_{1}\right|}{2}\right\rfloor\right)+\frac{\delta\left(\left|L_{1}\right|\right)}{2+c_{2}} \\
& \leq \frac{1}{2}\left(\left|L_{0}\right|+1+\frac{\left|L_{1}\right|-1}{2}\right)+\frac{1}{3} \\
& \leq \frac{1}{2}\left(\lfloor\tilde{\gamma}\rfloor+\frac{\lfloor 2 \tilde{\gamma}\rfloor-2}{2}\right)+\frac{1}{3} \leq \tilde{\gamma}-\frac{1}{2}+\frac{1}{3}<\tilde{\gamma} .
\end{aligned}
$$


If $\left|L_{1}\right| \leq\lfloor 2 \tilde{\gamma}\rfloor-3$, then we have

$$
\begin{aligned}
\tilde{u}_{1} & \leq \frac{1}{2}\left(\left|L_{0}\right|+1+\frac{\left|L_{1}\right|-1}{2}\right)+\frac{1}{3} \\
& \leq \frac{1}{2}\left(\lfloor\tilde{\gamma}\rfloor+1+\frac{\lfloor 2 \tilde{\gamma}\rfloor-4}{2}\right)+\frac{1}{3} \leq \tilde{\gamma}-\frac{1}{2}+\frac{1}{3}<\tilde{\gamma}
\end{aligned}
$$

Both cases contradict the minimality of $\tilde{\gamma}$.

(iii) Consider a strategy profile satisfying (V2) and (V6). Suppose that $c_{1}=1$ and $c_{2} \geq 2$. Since $\left|L_{1}\right|=0$ from (V6) and $\ell \geq 3$ from the assumption, we have

$$
\begin{aligned}
\tilde{u}_{2} & \leq \frac{1}{2}\left(1+\left\lfloor\frac{\left|L_{2}\right|}{2}\right\rfloor\right)+\frac{\delta\left(\left|L_{2}\right|\right)}{2+c_{3}} \\
& \leq \frac{1}{2}\left(1+\frac{\left|L_{2}\right|-1}{2}\right)+\frac{1}{3} \\
& \leq \frac{1}{2}\left(1+\frac{\lfloor 2 \tilde{\gamma}\rfloor-2}{2}\right)+\frac{1}{3} \leq \frac{\tilde{\gamma}}{2}+\frac{1}{3} .
\end{aligned}
$$

Since $\tilde{\gamma} \geq 1$, we obtain $\tilde{u}_{2}<\tilde{\gamma}$, which contradicts the minimality of $\tilde{\gamma}$.

Note that the assumption $\ell \geq 3$ in (iii) of Lemma 5 is needed because the strategy profile $\boldsymbol{\theta}=(2,3)$ and $\boldsymbol{c}=(1,2)$ on the path graph with 4 vertices will be a counterexample without it.

We now exhibit pure Nash equilibrium strategy profiles for $k$ players. It is trivial that the strategy profile given by

$$
\theta_{j}=j \text { and } c_{j}=1, \quad \forall j=1, \ldots, k
$$

on the path graph with $k$ vertices is a pure Nash equilibrium. First, we provide constructions of pure Nash equilibria for the path graph having an appropriate number of vertices in the following several lemmas.

Lemma 6 Suppose that there are $k$ players, where $k \geq 4$. Let $r$ be a positive integer greater than or equal to 2 , and $b_{j}$ a nonnegative integer less than or equal to $2 r-1$ for any $j=1,2, \ldots,\left\lfloor\frac{k-4}{2}\right\rfloor$. In addition, define $b_{\frac{k-3}{2}}=2 r-2$ when $k$ is odd. The strategy profile given by

$$
\left|L_{j}\right|= \begin{cases}r-1 & (j=0, k) \\ 0 & (j=1, k-1) \\ 2 r-2 & (2 \leq j \leq k-2, j: \text { even }) \\ b_{\frac{j-1}{2}} & (3 \leq j \leq k-2, j: \text { odd })\end{cases}
$$

and $c_{j}=1$ for $j=1, \ldots, k$ is a pure Nash equilibrium for the discrete Voronoi game on the path graph, if $b_{j-1}<2 r-1$ and $b_{j+1}<2 r-1$ hold for any $j$ with $b_{j}=0$ (see Figure 4).

Proof. It is obvious that $\tilde{\gamma}=r$. We can check that this strategy profile satisfies (V1)-(V6).

Lemma 6 implies that there exists a pure Nash equilibrium for $k$ players on the path graph with at least $2 r\left\lceil\frac{k}{2}\right\rceil-\delta(k)$ and at most $2 r\left\lceil\frac{k}{2}\right\rceil+\left\lfloor\frac{k-4}{2}\right\rfloor(2 r-1)-\delta(k)$ vertices, for any positive integer $r(\geq 2)$. 


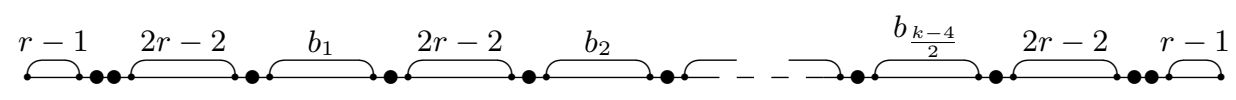

(a) $k$ is even

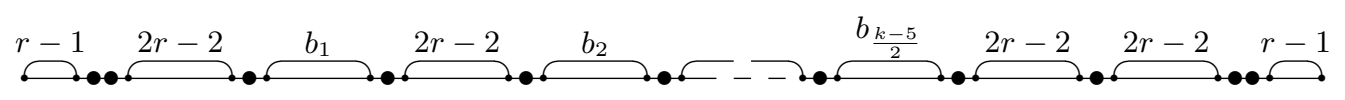

(b) $k$ is odd

Figure 4: Strategy profiles derived from (2).

Lemma 7 Assume there are $k$ players, where $k \geq 4$, and $p$ is an integer satisfying $2 \leq$ $p \leq k-2$. For a positive integer $r(\geq 2)$, the strategy profile given by

$$
\left|L_{j}\right|= \begin{cases}r-1 & (j=0, k) \\ 0 & (\langle 1 \leq j \leq k-3, j: \text { odd }\rangle \vee j=k-1) \\ 2 r-3 & (2 \leq j \leq p, j: \text { even }) \\ 2 r-2 & \text { (otherwise) }\end{cases}
$$

with $c_{j}=1$ for $j=1, \ldots, k$ is a pure Nash equilibrium for the discrete Voronoi game on the path graph (see Figure 5).

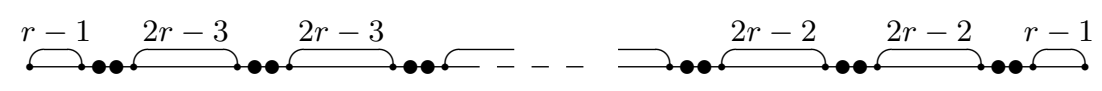

(a) $k$ is even

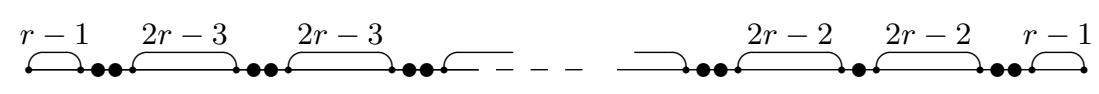

(b) $k$ is odd

Figure 5: Strategy profiles derived from (3).

Proof. It is obvious that $\tilde{\gamma}=r-\frac{1}{2}$. We can check that this strategy profile satisfies (V1)-(V6).

Lemma 7 implies that there exists a pure Nash equilibrium for $k$ players on the path graph with at least $2 r\left\lceil\frac{k}{2}\right\rceil-\left\lfloor\frac{k-2}{2}\right\rfloor-\delta(k)$ and at most $2 r\left\lceil\frac{k}{2}\right\rceil-1-\delta(k)$ vertices, for any positive integer $r(\geq 2)$.

Lemma 8 (i) On the path graph having at least $\frac{3 k+2}{2}$ vertices, the discrete Voronoi game with $k$ players admits a pure Nash equilibrium, if $k$ is even and greater than or equal to 6 . (ii) On the path graph having at least $\frac{3 k+5}{2}$ vertices, the discrete Voronoi game with $k$ players admits a pure Nash equilibrium, if $k$ is odd and greater than or equal to 9. 
Proof. Let $L B(k, r)=2 r\left\lceil\frac{k}{2}\right\rceil-\left\lfloor\frac{k-2}{2}\right\rfloor-\delta(k)$ and $U B(k, r)=2 r\left\lceil\frac{k}{2}\right\rceil+\left\lfloor\frac{k-4}{2}\right\rfloor(2 r-1)-\delta(k)$. Lemmas 6 and 7 imply that a pure Nash equilibrium exists for every path whose number of vertices lies in the interval $[L B(k, r), U B(k, r)]$ for some positive integer $r(\geq 2)$.

If $k$ is even, $L B(k, r)=r k-\frac{k-2}{2}$ and $U B(k, r)=r k+\frac{k-4}{2}(2 r-1)$. When $k \geq 6$, then we have $L B(k, r+1)-U B(k, r)=(1-r) k+4 r-1 \leq 1$. Thus, the strategy profiles obtained from (2) and (3) give pure Nash equilibria for all the path graphs with at least $\frac{3 k+2}{2}$ vertices for even $k(\geq 6)$ players.

If $k$ is odd, $L B(k, r)=r(k+1)-\frac{k-1}{2}$ and $U B(k, r)=r(2 k-4)-\frac{k-3}{2}$. When $k \geq 9$, then we have $L B(k, r+1)-U B(k, r)=(1-r) k+5 r \leq 1$. Thus, these strategy profiles give pure Nash equilibria for all the path graphs with at least $\frac{3 k+5}{2}$ vertices for odd $k(\geq 9)$ players.

When $k=2$, every path graph has a pure Nash equilibrium such as

$$
\theta_{1}=\left\lceil\frac{n}{2}\right\rceil, \text { and } c_{1}=2,
$$

where $n$ is the number of vertices. When $k=4$, for a positive integer $r$, the strategy profile given by the pair

$$
\boldsymbol{\theta}=(r, r+1, n-r, n-r+1) \text { and } \boldsymbol{c}=(1,1,1,1)
$$

is a pure Nash equilibrium on the path graph with $n=4 r$ or $n=4 r-1$ vertices. The strategy profile given by the pair

$$
\boldsymbol{\theta}=(r, n-r+1) \text { and } \boldsymbol{c}=(2,2)
$$

is also a pure Nash equilibrium on the path graph with $n=4 r-2$ or $n=4 r-3$.

Moreover, when $k$ is even, we can verify that, for the path graph with $\frac{3 k}{2}$ vertices, the strategy profile given by

$$
\theta_{j}=3 j-1, \text { and } c_{j}=2, \quad \forall j=1, \ldots, \frac{k}{2}
$$

is a pure Nash equilibrium (see (a) in Figure 6), and for the path graph with $\frac{3 k-4}{2}$ vertices, by

$$
\begin{gathered}
\theta_{1}=2, \theta_{j}= \begin{cases}\theta_{j-1}+1 & (2 \leq j \leq k-2, j: \text { even }) \\
\theta_{j-1}+2 & (3 \leq j \leq k-3, j: \text { odd }),\end{cases} \\
c_{1}=c_{k-2}=2, \text { and } c_{2}=c_{3}=\cdots=c_{k-3}=1
\end{gathered}
$$

is a pure Nash equilibrium (see (b) in Figure 6). In addition, it is obvious that

$$
\theta_{j}=2 j \text { and } c_{j}=2, \quad \forall j=1, \ldots, \frac{k}{2}
$$

is also pure Nash equilibrium on the path graph with $k+1$ vertices (see (c) in Figure 6).

From these examples of strategy profiles, together with Lemma 8, we obtain the following Theorem.

Theorem 9 Suppose that $k$ is even. If $n=k, n=k+1, n=\frac{3 k-4}{2}$ or $n \geq \frac{3 k}{2}$, then the discrete Voronoi game with $k$ players on the path graph with $n$ vertices admits a pure Nash equilibrium. 


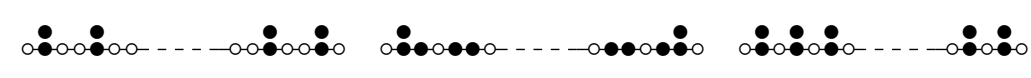

(a) (b) (c)

Figure 6: Pure Nash equilibria for discrete Voronoi games for even players.

We now turn to the case of odd players. Assume that $k \geq 5$. For the path graph with $\frac{3 k+3}{2}$ vertices, we can verify that the strategy profile given by

$$
\theta_{j}=3 j-1 \quad \forall j=1, \ldots, \frac{k+1}{2}, \quad \text { and } \quad c_{j}= \begin{cases}1 & (j=2) \\ 2 & (j \neq 2)\end{cases}
$$

is a pure Nash equilibrium (see (a) in Figure 7). For the path graph with $\frac{3 k-1}{2}$ vertices, we can also confirm that the strategy profile given by

$$
\begin{gathered}
\theta_{1}=2, \theta_{j}=\left\{\begin{array}{cc}
\theta_{j-1}+1 & (2 \leq j \leq k-1, j: \text { even }) \\
\theta_{j-1}+2 & (3 \leq j \leq k-1, j: \text { odd }),
\end{array}\right. \\
c_{1}=2, \text { and } c_{2}=c_{3}=\cdots=c_{k-1}=1
\end{gathered}
$$

is a pure Nash equilibrium (see (b) in Figure 7). Moreover, it is obvious that

$$
\theta_{j}=\left\{\begin{array}{ll}
2 j & \left(j=1, \ldots, \frac{k-1}{2}\right) \\
k & \left(j=\frac{k+1}{2}\right)
\end{array} \quad \text { and } c_{j}= \begin{cases}1 & \left(j=\frac{k-1}{2}\right) \\
2 & \left(j \neq \frac{k-1}{2}\right)\end{cases}\right.
$$

on the path graph with $k+1$ vertices is also a pure Nash equilibrium (see (c) in Figure 7).

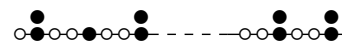

(a) (b)

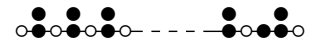

(c)

Figure 7: Pure Nash equilibria for discrete Voronoi games for odd players.

For $k=7$, a similar argument as the proof of Lemma 8 shows that $L B(7, r+1)-$ $U B(7, r) \leq 1$ holds for $r \geq 3$. Since $L B(7,3)=21$ and $U B(7,2)=18$, we can construct a pure Nash equilibrium from $(2)$ and (3) on the path graph with at least $\frac{3 k+5}{2}$ vertices except 19 and 20 vertices. For the path graphs with 19 and 20 vertices, the strategy profiles given by

$$
\boldsymbol{\theta}=(3,4,8,11,14,17,18) \text { and } \boldsymbol{c}=(1,1,1,1,1,1,1)
$$

and by

$$
\boldsymbol{\theta}=(3,4,8,11,15,18) \text { and } \boldsymbol{c}=(1,1,1,1,1,2)
$$

are pure Nash equilibria, respectively (see Figure 8 ).

The above discussion concludes the following theorem. 


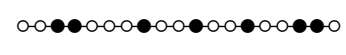

$n=19$

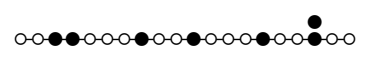

$n=20$

Figure 8: Pure Nash equilibria for discrete Voronoi games with 7 players on the path with 19 and 20 vertices.

Theorem 10 Suppose that $k$ is odd and $k \geq 7$. If $n=k, n=k+1, n=\frac{3 k-1}{2}$ or $n \geq \frac{3 k+3}{2}$, then the discrete Voronoi game with $k$ players on the path graph with $n$ vertices admits a pure Nash equilibrium.

Now, we address to the cases of $k=3$ and $k=5$, which are not mentioned in Theorem 10.

Lemma 11 The discrete Voronoi game with 3 players on the path graph with $n$ vertices possesses a pure Nash equilibrium, if and only if $n \leq 4$.

Proof. Since we know that there exists a pure Nash equilibrium for $n \leq 3$, consider the case of $n \geq 4$. If there exists a pure Nash equilibrium with $c_{1}=c_{2}=c_{3}=1$, then (V6) derives $\left|L_{1}\right|=\left|L_{2}\right|=0$, which implies that $\left|L_{0}\right|=\left\lfloor\frac{\left|L_{2}\right|+1}{2}\right\rfloor=0$ and $\left|L_{3}\right|=\left\lfloor\frac{\left|L_{1}\right|+1}{2}\right\rfloor=0$. Thus, it is possible only when $n=k$. If a pure Nash equilibrium with $c_{1}=1$ and $c_{2}=2$ exists, we have $\tilde{u}_{2}=\frac{\left|L_{2}\right|+1}{2}$ since $\left|L_{1}\right|=0$. A utility of a player on $\theta_{2}$ becomes $\left|L_{2}\right|$, if he/she moves to $\theta_{2}+1$. Because this utility is not greater than $\tilde{u}_{2}$, we have $\left|L_{2}\right| \leq 1$. Hence, we obtain $\left|L_{0}\right| \leq 1$ and $n \leq 4$.

Lemma 12 The discrete Voronoi game with 5 players on the path with $n$ vertices possesses a pure Nash equilibrium, if and only if $n \not \equiv 2(\bmod 6)$.

Proof. We enumerate all strategy profiles satisfying (V1)-(V6).

First, we consider pure Nash equilibria with $c_{j}=1$ for $j=1, \ldots, 5$. It follows from (V6) that $\left|L_{1}\right|=\left|L_{4}\right|=0$. If $\left|L_{0}\right|=\left|L_{5}\right|=r-1$, then (V6) implies that each of $\left|L_{2}\right|$ and $\left|L_{3}\right|$ is either $2 r-2$ or $2 r-3$. These strategy profiles satisfy (V1)-(V6). Thus, these give pure Nash equilibria on the path graphs with $6 r-1,6 r-2$ and $6 r-3$, respectively (see (a), (b) and (c) in Figure 9). If $\left|L_{0}\right|>\left|L_{5}\right|=r-1$, then $\left|L_{0}\right|=r$ and $\left|L_{2}\right|=2 r-1$ from (V2) and (V6), since $\tilde{\gamma} \leq \tilde{u}_{5}=r$. If $\left|L_{3}\right| \leq 2 r-3$, then the utility $\tilde{u}_{4}$ is less than $r$, which contradicts $\left|L_{0}\right|=r \leq\lfloor\tilde{\gamma}\rfloor \leq \tilde{u}_{4}$. Thus, $\left|L_{3}\right|=2 r-2$. In this case, (V5) is not satisfied since $\tilde{\gamma}=r$.

Next, we discuss the case where $c_{j}=2$ for some $j$. From the condition (iii) in Lemma 5 , such $j$ is only either 1 or $\ell$. When $c_{1}=c_{3}=2$ and $c_{2}=1$, the condition (ii) in Lemma 5 implies that $\left|L_{0}\right|=\left|L_{3}\right|=\lfloor\tilde{\gamma}\rfloor$. Suppose that $\left|L_{0}\right|=\left|L_{3}\right|=r$. Since $2 r \leq\lfloor 2 \tilde{\gamma}\rfloor \leq 2 r+1$, each of $\left|L_{1}\right|$ and $\left|L_{2}\right|$ is either $2 r, 2 r-1$ or $2 r-2$. Without loss of generality, we assume that $\left|L_{1}\right| \leq\left|L_{2}\right|$. When $\left|L_{2}\right|=2 r$, then it follows from (V2) that $\tilde{\gamma} \geq r+\frac{1}{2}$. Thus, to satisfy $\tilde{u}_{1} \geq r+\frac{1}{2}$, we have $\left|L_{1}\right|=2 r$. This strategy profile gives a pure Nash equilibrium on the path with $6 r+3$ vertices (see (d) in Figure 9). When $\left|L_{2}\right|=2 r-1$, then it violates (V3) if $\left|L_{1}\right|=\left|L_{2}\right|=2 r-1$. Thus, we have $\left|L_{1}\right|=2 r-2$. The rest possibility is $\left|L_{1}\right|=\left|L_{2}\right|=2 r-2$. These also satisfy (V1)-(V6) and give pure Nash equilibria on 
path graphs with $6 r$ and $6 r-1$ vertices (see (e) and (f) in Figure 9). Finally, we treat pure Nash equilibria with $c_{1}=2$ and $c_{2}=c_{3}=c_{4}=1$, in which $\left|L_{3}\right|=0$. Suppose that $\left|L_{0}\right|=r=\lfloor\tilde{\gamma}\rfloor$. The condition (ii) in Lemma 5, (V2) and (V3) imply that $\left|L_{1}\right|$ is either $2 r-2$ or $2 r$. Moreover, $\left|L_{2}\right| \geq 2 r-2$ and $\left|L_{4}\right| \geq r-1$ hold, since $\tilde{\gamma} \geq r$. If $\left|L_{4}\right|=r-1$, then $\left|L_{2}\right|=2 r-2$ from (V6). In addition, $\left|L_{1}\right|=2 r-2$ from (V2) since $\tilde{\gamma} \leq r$. This strategy profile is a pure Nash equilibrium on the path graph with $6 r-1$ vertices (see (g) in Figure 9). If $\left|L_{4}\right|=r$, then $\left|L_{2}\right|=2 r-1$ or $2 r$ from (V6). In this case, there are three possible strategy profiles shown in (h) (i) and (j) in Figure 9. If $\left|L_{1}\right|=2 r-2$, then $\tilde{u}_{1}=r \geq \tilde{\gamma}$, which implies that $\left|L_{2}\right| \leq 2 r-1$ from (V2). If $\left|L_{1}\right|=2 r$, then $\left|L_{2}\right|$ can be $2 r-1$ or $2 r$. These strategy profiles are also pure Nash equilibria on path graphs with $6 r+1,6 r+3$ and $6 r+4$, respectively.

We complete the enumeration of all possibility, and obtain pure Nash equilibria on the path graphs with $6 r, 6 r+1,6 r+3,6 r+4$, and $6 r+5$, respectively.

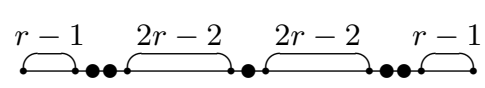

(a): $\tilde{\gamma}=r$

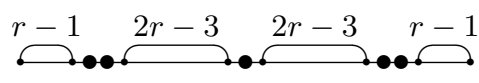

(c): $\tilde{\gamma}=r-\frac{1}{2}$

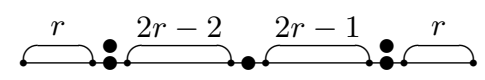

(e): $\tilde{\gamma}=r$

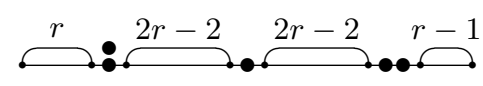

$(\mathrm{g}): \tilde{\gamma}=r$

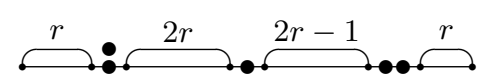

(i): $\tilde{\gamma}=r+\frac{1}{2}$

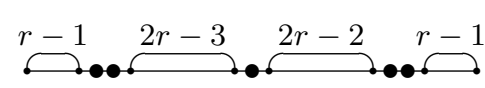

(b): $\tilde{\gamma}=r-\frac{1}{2}$

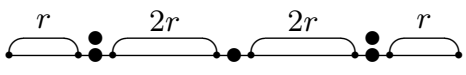

(d): $\tilde{\gamma}=r+\frac{1}{2}$

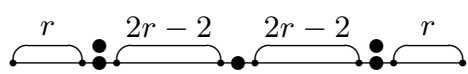

(f): $\tilde{\gamma}=r$

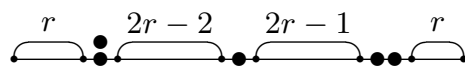

(h): $\tilde{\gamma}=r$

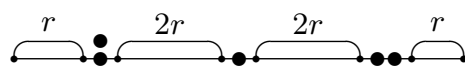

$(\mathrm{j}): \tilde{\gamma}=r+\frac{1}{2}$

Figure 9: All pure Nash equilibria for the discrete Voronoi game with 5 players on the path graphs.

Lemmas 11 and 12 are necessary and sufficient conditions for the existence of pure Nash equilibria in path graphs for 3 and 5 players. On the other hand, Theorems 9 and 10 only claim the conditions in the statements are necessary for the existence of pure Nash equilibria in the path graphs except $k=3,5$. We show these conditions are also sufficient in the following theorem.

Theorem 13 (i) If $k$ is even, the discrete Voronoi game with $k$ players on the path graph with $n$ vertices does not admit pure Nash equilibria for $k+2 \leq n \leq \frac{3 k-6}{2}$ or $n=\frac{3 k-2}{2}$.

(ii) If $k$ is odd with $k \geq 7$, the discrete Voronoi game with $k$ players on the path graph with $n$ vertices does not admit pure Nash equilibria for $k+2 \leq n \leq \frac{3 k-3}{2}$ or $n=\frac{3 k+1}{2}$. 
Proof. We first consider Nash equilibria with $1 \leq \tilde{\gamma}<\frac{3}{2}$. Since $\lfloor\tilde{\gamma}\rfloor=1$ and $\lfloor 2 \tilde{\gamma}\rfloor=2$, (V2) derives $\left|L_{j}\right| \leq 1$ for all $j=0, \ldots, \ell$.

When $c_{j}=1$ for all $j=1, \ldots, \ell$, we have $\left|L_{1}\right|=\left|L_{\ell-1}\right|=0$ from (V6). If $\left|L_{0}\right|=0$, then (V6) implies that $\left|L_{2}\right|=0$. By applying (V5) inductively, we have $\left|L_{j}\right|=0$ for all $j=3, \ldots, \ell$, which coincides with the strategy profile by (1). If $\left|L_{0}\right|=1$, then (V6) implies that $\left|L_{2}\right|=1$. By applying (V4) and (V5) inductively, we have

$$
\left|L_{i}\right|= \begin{cases}1 & (j: \text { even }) \\ 0 & (j: \text { odd })\end{cases}
$$

However, we obtain $\tilde{\gamma}=\frac{3}{2}$ in this strategy profile. Thus, the assumption is not satisfied.

When there exists some $j$ with $2 \leq j \leq \ell-1$ such that $c_{j}=2$, the condition (i) in Lemma 5 implies that $\left|L_{j-1}\right|=\left|L_{j}\right|=1$. In order to satisfy (V3), $c_{j}=2$ for all $j$ except $j=1, \ell-1$. Thus, admitted strategy profiles are given by $(5),(7)$, or

$$
\theta_{1}=2, \theta_{j}=\left\{\begin{array}{ll}
\theta_{j-1}+2 & (j \neq 2, \ell) \\
\theta_{j-1}+1 & (j=2, \ell)
\end{array} \text { and } c_{j}= \begin{cases}2 & (j \neq 2, \ell-1) \\
1 & (j=2, \ell-1)\end{cases}\right.
$$

on the path graph with $k+1$ vertices. When $c_{1}=2$ and $c_{2}=\cdots=c_{\ell-1}=1$, we have $\left|L_{0}\right|=1$ and $\left|L_{1}\right|=0$ from the condition (ii) in Lemma 5 and (V3). If $\left|L_{2}\right|=0$, by applying (V5) inductively, we have $\left|L_{3}\right|=\left|L_{4}\right|=\cdots=\left|L_{\ell-1}\right|=0$. In this case, if $c_{\ell}=1$, then $\left|L_{\ell}\right|=\left\lfloor\frac{2\left|L_{\ell-2}\right|+1}{2}\right\rfloor=0$ from (V6). And if $c_{\ell}=2$, then $\left|L_{\ell}\right|=1$ from the condition (ii) in Lemma 5. Thus, we obtain $n=k$ irrespective of $c_{\ell}$. If $\left|L_{2}\right|=1$, by applying (V4) and (V5) inductively, we have

$$
\left|L_{i}\right|= \begin{cases}1 & (j: \text { even }) \\ 0 & (j: \text { odd })\end{cases}
$$

Such a strategy profile is obtained from (4) or (6). Here (4) implies $k$ is even and $n=\frac{3 k-4}{2}$, and (6) implies $k$ is odd and $n=\frac{3 k-1}{2}$.

The discussion above covers the cases with $n<\frac{3 k}{2}$, since we have $\tilde{\gamma} \leq \frac{n}{k}<\frac{3}{2}$. Thus, what remains is the case that $k(\geq 7)$ is odd and $n=\frac{3 k+1}{2}$. Suppose that there exists a pure Nash equilibrium. Let $k$ be the smallest number of players such that a pure Nash equilibrium exists on the path graph with $\frac{3 k+1}{2}$ vertices. From Lemmas 11 and 12 , we know $k \geq 7$. Because of the former discussion in this proof, we have $\tilde{\gamma} \geq \frac{3}{2}$, which implies that $\frac{3}{2} \leq \tilde{u}_{j} \leq 2$ for all $j=1, \ldots, \ell$. Moreover, if $\tilde{u}_{j}=2$ for some $j$, then the rest of utilities $\tilde{u}_{j^{\prime}}$ with $j^{\prime} \neq j$ is equal to $\frac{3}{2}$. If $c_{1}=1$, then $\left|L_{1}\right|=0$ and $\tilde{u}_{1}=\left|L_{0}\right|+1$. In this case, we have $\tilde{u}_{1}=2$. In addition, (iii) in Lemma 5 implies that $c_{2}=1$. Since $\tilde{u}_{2}=\frac{3}{2}$, we have $\left|L_{2}\right|=1$ and $c_{3}=1$. By the same way, $\left|L_{3}\right|=0$ holds. Since $\tilde{\gamma}=\frac{3}{2}$, we have $\left|L_{4}\right| \leq 2$ from (V2). Thus, we obtain $c_{4}=1$, because if not, $\tilde{u}_{4} \leq \frac{1}{2}\left(1+\frac{\left|L_{4}\right|}{2}\right)<\frac{3}{2}$. Hence, if we remove vertices between $\theta_{2}$ and $\theta_{3}$, that is, $\{3,4,5\}$, from this path graph, then the resulting strategy profile is a Nash equilibrium for $(k-2)$ players on the path graph with $\frac{3 k+1}{2}-3=\frac{3(k-2)+1}{2}$. If $c_{1}=2$, then $\left|L_{0}\right|=1$ from the condition (ii) in Lemma 5. Moreover $\left|L_{1}\right|=2$ since $\tilde{u}_{1} \geq \frac{3}{2}$. When $c_{2}=2$, then $\left|L_{2}\right|=2$ is obtain by the same way. When $c_{2}=1$, then $\left|L_{2}\right|=0$ since $\tilde{u}_{2} \leq 2$. Hence, if we remove vertices $\{1,2,3\}$ from this path graph, then the resulting strategy profile is a Nash equilibrium for $(k-2)$ players on a path with $\frac{3(k-2)+1}{2}$. In both cases, we have a contradiction for the minimality of $k$. 
We summarize the existence of pure Nash equilibria for the discrete Voronoi games with $k$ players in Table 1 . Particularly, Table 2 shows for small $k$ and $n$. In these tables, " $\checkmark "$ and "-" mean that existence and nonexistence of pure Nash equilibria, respectively. It is interesting that the games on path graphs are more complicated than on cycle graphs.

Table 1: The existence of a pure Nash equilibria for the discrete Voronoi games.

\begin{tabular}{|c|c|c|c|c|c|c|c|}
\hline$n$ & $k$ & $k+1$ & $k+2 \leq \cdots \leq\left\lceil\frac{3 k}{2}\right\rceil-3$ & $\left.\frac{3 k}{2}\right\rceil-2$ & $\left\lceil\frac{3 k}{2}\right\rceil-1$ & $\left.\frac{3 k}{2}\right\rceil$ & $\left.\frac{3 k}{2}\right\rceil+1 \leq \cdots$ \\
\hline cycle $[3,7]$ & $\checkmark$ & - & - & - & - & $\checkmark$ & $\checkmark$ \\
\hline path $\quad(k:$ even $)$ & $\checkmark$ & $\checkmark$ & - & $\checkmark$ & - & $\checkmark$ & $\checkmark$ \\
\hline$(k \geq 7$, odd $)$ & $\checkmark$ & $\checkmark$ & - & - & $\checkmark$ & - & $\checkmark$ \\
\hline
\end{tabular}

Table 2: The existence of a pure Nash equilibria for discrete the Voronoi games on a path graph for small $k$ and $n$.

\begin{tabular}{|cc||ccccccccccccccccc}
\hline$k$ & & $n$ & 2 & 3 & 4 & 5 & 6 & 7 & 8 & 9 & 10 & 11 & 12 & 13 & 14 & 15 & 16 & $\ldots$ \\
\hline 2 & $\checkmark$ & $\checkmark$ & $\checkmark$ & $\checkmark$ & $\checkmark$ & $\checkmark$ & $\checkmark$ & $\checkmark$ & $\checkmark$ & $\checkmark$ & $\checkmark$ & $\checkmark$ & $\checkmark$ & $\checkmark$ & $\checkmark$ & $\checkmark$ \\
3 & & & $\checkmark$ & $\checkmark$ & - & - & - & - & - & - & - & - & - & - & - & - & - \\
4 & & & $\checkmark$ & $\checkmark$ & $\checkmark$ & $\checkmark$ & $\checkmark$ & $\checkmark$ & $\checkmark$ & $\checkmark$ & $\checkmark$ & $\checkmark$ & $\checkmark$ & $\checkmark$ & $\checkmark$ & $\checkmark$ \\
5 & & & & $\checkmark$ & $\checkmark$ & $\checkmark$ & - & $\checkmark$ & $\checkmark$ & $\checkmark$ & $\checkmark$ & $\checkmark$ & - & $\checkmark$ & $\checkmark$ & $\checkmark$ \\
6 & & & & & $\checkmark$ & $\checkmark$ & - & $\checkmark$ & $\checkmark$ & $\checkmark$ & $\checkmark$ & $\checkmark$ & $\checkmark$ & $\checkmark$ & $\checkmark$ & $\checkmark$ \\
7 & & & & & $\checkmark$ & $\checkmark$ & $\checkmark$ & - & $\checkmark$ & - & $\checkmark$ & $\checkmark$ & $\checkmark$ & $\checkmark$ & $\checkmark$ & $\checkmark$ \\
8 & & & & & & $\checkmark$ & $\checkmark$ & $\checkmark$ & $\checkmark$ & - & $\checkmark$ & $\checkmark$ & $\checkmark$ & $\checkmark$ & $\checkmark$ & $\checkmark$ \\
9 & & & & & & & $\checkmark$ & $\checkmark$ & $\checkmark$ & - & $\checkmark$ & $\checkmark$ & \\
10 & & & & & & & & $\checkmark$ & $\checkmark$ & - & - & $\checkmark$ & - & $\checkmark$ & $\checkmark$ & $\checkmark$ \\
& & & & & & & & & $\checkmark$ & $\checkmark$ & - & $\checkmark$ & - & $\checkmark$ & $\checkmark$ & $\checkmark$
\end{tabular}

Remark 1 The strategy profiles given by (2) and (3) are pure Nash equilibria on cycle graphs by adding an edge $(1, n)$ to the path graph. Mavronicolas et al. [7] have shown pure Nash equilibria on cycle graphs by only standard strategy profiles in which $\left|L_{i}\right|$ is given by $\left\lfloor\frac{k}{n-k}\right\rfloor$ or $\left\lceil\frac{k}{n-k}\right\rceil$. On the other hand, our strategy profiles are not standard. Thus, our examples demonstrate variety of pure Nash equilibria.

Remark 2 We call a strategy profile simple if $c_{j}=1$ for all $j=1, \ldots, \ell$. As discussed in the next section, pure Nash equilibria are necessarily simple for information diffusion games. On the other hand, in discrete Voronoi games pure Nash equilibria can be nonsimple. This difference is one of the causes of the different behavior of pure Nash equilibria between discrete Voronoi games and information diffusion games. When $k=2$ and $n$ is odd, the unique pure Nash equilibrium is given by a nonsimple strategy profile. Also, when $k=4$ and $n \equiv 2(\bmod 4)$ or $\equiv 3(\bmod 4)$, there are no simple strategy profile giving pure Nash equilibria. 


\subsection{Information diffusion game}

In the path graph, the analysis of pure Nash equilibria of information diffusion games is simpler than that of discrete Voronoi games. In information diffusion games, the utility of the player who chooses the vertex $\theta_{j}$ becomes zero if $c_{j} \geq 2$. Hence, we only need to consider strategy profiles with $c_{j}=1$ for all $j$, namely, simple strategy profiles. Thus, we express the strategy profiles only by $\boldsymbol{\theta}$ instead of $(\boldsymbol{\theta}, \boldsymbol{c})$. Note that the number of vertices chosen by the players in a simple strategy profile equals the number of the players, that is, $\ell=k$. The utility of the player who chooses the vertex $\theta_{j}$ is given by

$$
u_{j}(\boldsymbol{\theta})= \begin{cases}\left|L_{0}\right|+\left\lfloor\frac{\left|L_{1}\right|}{2}\right\rfloor+1 & (j=1) \\ \left\lfloor\frac{\left|L_{j-1}\right|}{2}\right\rfloor+\left\lfloor\frac{\left|L_{j}\right|}{2}\right\rfloor+1 & (1<j<k) \\ \left\lfloor\frac{\left|L_{k-1}\right|}{2}\right\rfloor+\left|L_{k}\right|+1 & (j=k) .\end{cases}
$$

For short, we write $u_{j}$ instead of $u_{j}(\boldsymbol{\theta})$.

The following theorem characterizes the existence of pure Nash equilibria for the information diffusion game on a path graph.

Theorem 14 For the information diffusion game with $k$ players on the path graph with $n(\geq k)$ vertices, a strategy profile defined by $\boldsymbol{\theta}$ is a pure Nash equilibrium if and only if the following hold:

(I1) $\left|L_{j}\right| \leq 2 \gamma$ for all $j=1, \ldots, k-1,\left|L_{0}\right| \leq \gamma$ and $\left|L_{k}\right| \leq \gamma$.

(I2) Both of $\left|L_{j-1}\right|$ and $\left|L_{j}\right|$ are not simultaneously odd. Namely, $\delta\left(\left|L_{j-1}\right|\right)+\delta\left(\left|L_{j}\right|\right) \leq 1$ for $j=2, \ldots, k-1$.

(I3) $\left|L_{1}\right|=0$ and $\left|L_{k-1}\right|=0$.

Here, $\gamma=\min \left\{u_{j} \mid j=1, \ldots, k\right\}$ is the minimum utility.

Proof. For $j=2, \ldots, k-1$, the player on $\theta_{j}$ cannot improve his utility by moving to any vertex in $L_{j-1} \cup L_{j}$ if and only if (I2) is satisfied. The players on $\theta_{1}$ and $\theta_{k}$ cannot improve their utility by moving to neighboring vertices, if and only if (I3) is satisfied. The player on $\theta_{j}$ cannot improve his/her utility by moving to any vertex in $L_{i}$ with $i \neq j-1$ and $i \neq j$, if and only if (I1) satisfied.

Lemma 15 On the path graph having more than six vertices, the information diffusion game with three players does not possess a pure Nash equilibrium.

Proof. It follows from (I3) that a pure Nash equilibrium satisfies $\theta_{2}=\theta_{1}+1$ and $\theta_{3}=\theta_{2}+1$. Thus, we have $\gamma=u_{2}=1$, which implies that $\left|L_{0}\right|$ and $\left|L_{3}\right|$ is at most 1 . Hence, if a strategy profile satisfies (I1)-(I3), then the number of vertices is at most 5 .

We now exhibit pure Nash equilibria for each $k$. When $k=2$, the only equilibrium is given by the paired location in center, similar to Hotelling's location games and discrete Voronoi games. Namely, $\left(\theta_{1}, \theta_{2}\right)$ given by either $\left(\left\lfloor\frac{n}{2}\right\rfloor,\left\lfloor\frac{n}{2}\right\rfloor+1\right)$ or $\left(\left\lceil\frac{n}{2}\right\rceil,\left\lceil\frac{n}{2}\right\rceil+1\right)$ is the desired location. When $k=4$, we can verify that strategy profiles $\left(\theta_{1}, \theta_{2}, \theta_{3}, \theta_{4}\right)$ given by $\left(\left\lfloor\frac{n}{4}\right\rfloor,\left\lfloor\frac{n}{4}\right\rfloor+1,\left\lfloor\frac{3 n}{4}\right\rfloor,\left\lfloor\frac{3 n}{4}\right\rfloor+1\right)$ and $\left(\left\lceil\frac{n}{4}\right\rceil,\left\lceil\frac{n}{4}\right\rceil+1,\left\lceil\frac{3 n}{4}\right\rceil,\left\lceil\frac{3 n}{4}\right\rceil+1\right)$ are pure Nash equilibria. Further, Figure 10 shows all Nash equilibria for information diffusion games with four 
when $n=4 r$

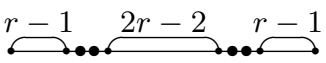

when $n=4 r+1$

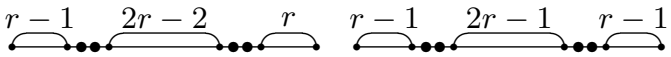

when $n=4 r+2$

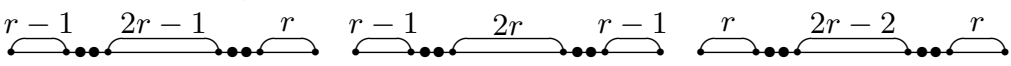

when $n=4 r+3$

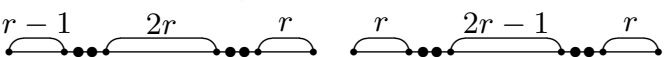

Figure 10: All Nash equilibria for the information diffusion game with four players on the path graph.

players, provided that $\left|L_{0}\right| \leq\left|L_{4}\right|$. All these strategy profiles satisfy $\gamma=r$. When $k=5$, unlike the case of the discrete Voronoi games, we can obtain pure Nash equilibria shown in Figure 11, where $r$ is a nonnegative integer. These strategy profiles also provide $\gamma=r$, and it is easy to verify these satisfy (I1)-(I3).

Theorem 16 The information diffusion game on the path graph possesses a pure Nash equilibrium when the number of player is not three.

Proof. Strategy profiles $\boldsymbol{\theta}$ given by (2) and (3), respectively, satisfy (I1)-(I3). Thus, these are pure Nash equilibria for both of discrete Voronoi games and information diffusion games, and the similar arguments of Lemma 8 also hold for the information diffusion games. Together with the case of $k=2$ and 4 described in the above of this Theorem, we have pure Nash equilibria on the path graph whose number of vertices is more than or equal to $\frac{3 k+2}{2}$ for even $k$. For odd $k$, we showed pure Nash equilibria for $k=5$. When $k=7$, a similar argument as the proof of Lemma 8 provides a pure Nash equilibrium from (2) and (3) on the path graph with at least $\frac{3 k+5}{2}$ vertices except 19 and 20 vertices. In addition, a strategy profile

$$
\boldsymbol{\theta}=(3,4,8,11,14,17,18)
$$

is a pure Nash equilibrium on the path graph with both base of 19 vertices and 20 vertices. Thus, for any $k$ except 3 , we obtain pure Nash equilibria for the information diffusion games on a path graph whose number of vertices is more than or equal to $\frac{3 k+2}{2}$ for even $k$ and $\frac{3 k+5}{2}$ for odd $k$.

Moreover, a strategy profile $\boldsymbol{\theta}^{\prime}$ given by

$$
\left|L_{j}\right|= \begin{cases}1 & (j: \text { even, } 0 \leq j \leq p) \\ 0 & \text { (otherwise) }\end{cases}
$$

satisfies (I1)-(I3), for any $p$ less than $k-1$. Additionally, a strategy profile obtained from $\boldsymbol{\theta}^{\prime}$ by replacing $\left|L_{k}\right|=1$ instead of $\left|L_{k}\right|=0$ is still a pure Nash equilibrium. Thus, on the 


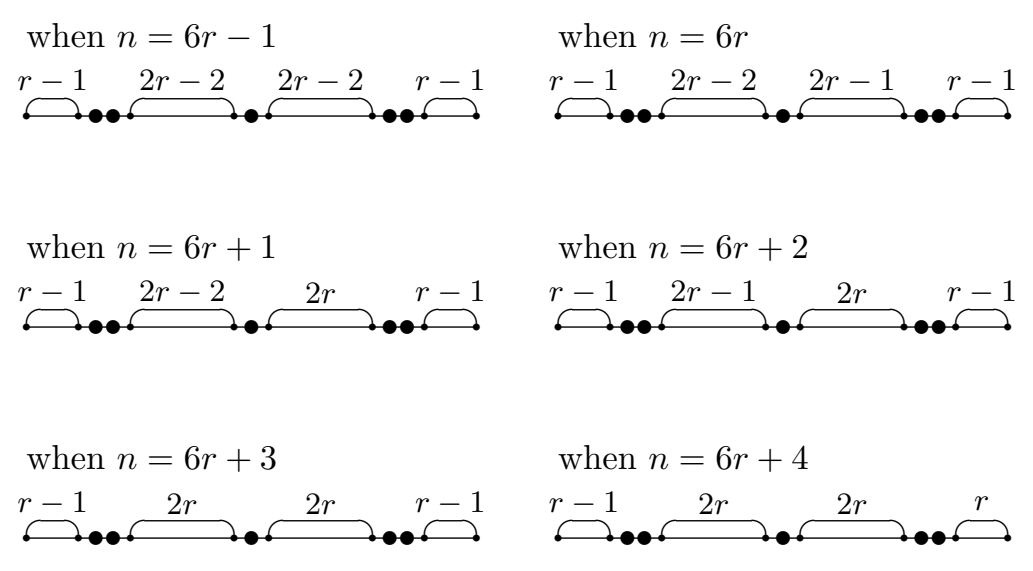

Figure 11: Pure Nash equilibria for the information diffusion game with five players on the path graph.

path graphs having less than $\left\lfloor\frac{3 k+3}{2}\right\rfloor$ vertices, the information diffusion games have pure Nash equilibria.

Therefore, we conclude the existence of pure Nash equilibria for any case except $k=3$.

Remark 3 By a similar way, we can give a characterization for the existence of pure Nash equilibria on cycle graphs. A strategy profile $\boldsymbol{\theta}$ is a pure Nash equilibrium if and only if

(I1)' $\left|L_{j}\right| \leq 2 \gamma$ for all $j$

(I2)' both of $\left|L_{j-1}\right|$ and $\left|L_{j}\right|$ are not simultaneously odd, for all $j$. Here, identify $L_{0}$ with $L_{k}$.

Therefore, on a cycle graph by adding an edge $(0, n)$ to the path graph, the strategy profiles discussed in the proof of Theorem 16, that is to say, given by $(2),(3),(8)$ and (9), satisfy (I1)' and (I2)'. Hence, these show that the information diffusion games on the cycle graphs have pure Nash equilibria for any number of players except three. On the other hand, for the games with three players on the cycle graphs, consider the strategy profiles as follows: $\boldsymbol{\theta}=\left(1,\left\lceil\frac{n}{2}\right\rceil, n\right)$ if $\left\lceil\frac{n}{2}\right\rceil$ is even, and $\boldsymbol{\theta}=\left(1,\left\lfloor\frac{n}{2}\right\rfloor, n\right)$ otherwise. These satisfy (I1)' and (I2)', and thus we see that the game on the cycle graphs has always pure Nash equilibria also for the case the number of players is three. (For the case the number of players is three, Ito and Muramatsu [6] have previously shown a partial result.)

\section{Discussion}

In the previous section, we gave the complete characterization for existence of pure Nash equilibria for both of the discrete Voronoi games and the information diffusion games on the path graphs and the cycle graphs. On tree graphs, Ito and Muramatsu [6] and Small 


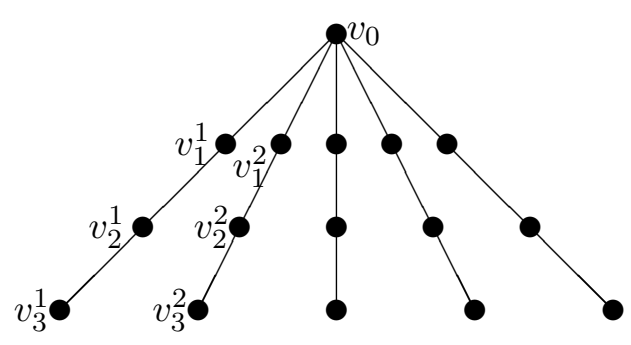

Figure 12: $(5,3)$-spider graph

and Mason [8] discussed the existence of pure Nash equilibria for the information diffusion games. For a tree graph, Small and Mason showed that a pure Nash equilibrium strategy profile is given by a pair of adjacent vertices $\boldsymbol{x}^{*}=(v, w)$ such that $\left|U_{1}(v, w)-U_{2}(v, w)\right|$ is minimized. Note that this strategy profile $\boldsymbol{x}^{*}$ is also a pure Nash equilibrium for the discrete Voronoi game if $U_{1}(v, w)=U_{2}(v, w)$. If $U_{1}(v, w) \neq U_{2}(v, w)$, without loss of generality, assume that $U_{1}(v, w)>U_{2}(v, w)$. Then, a strategy profile where the both players choose $v$ is a pure Nash equilibrium for the discrete Voronoi game.

We now show, for at least three players, there exists a tree on which any pure Nash equilibrium is not admitted. Our Lemmas 11 and 15 describe for three players on the path graphs, a special case of tree graphs. We generalize these results to spider graphs. We call a graph $(s, t)$-spider if it is obtained from the star $K_{1, s}$ by subdividing each edge by $t-1$ new vertices. Note that the $(s, t)$-spider graph has $s t+1$ vertices. Let $v_{0}$ be the vertex in the center, and we name other vertices as $v_{1}^{i}, v_{2}^{i}, \ldots, v_{t}^{i}$ along each $i$-th paths $(i=1, \ldots, s)$ from the vertex next to the center to the leaf. (See Figure 12.)

Lemma 17 On the $(k-1, t)$-spider graph for any $k \geq 3$ and $t \geq 3$, neither the discrete Voronoi game nor the information diffusion game with $k$ players possess a pure Nash equilibrium.

Proof. First, we observe two claims.

claim 1: If there exists $i$ such that no vertices of $\left\{v_{j}^{i} \mid j=1, \ldots, t\right\}$ are chosen by any players, then such a strategy profile is not a pure Nash equilibrium.

Proof of claim 1. Since players share the number of vertices as their utility, there exists a player whose utility is at most $\frac{t(k-1)+1}{k}<t$. If this player relocates into $v_{1}^{i}$, then his/her utility increases to $t$.

The properties in the next claim can be shown by arguments similar to our previous discussion around the conditions (V6) and (I3). For convenience, we represent $v_{0}$ as $v_{0}^{i}$ for each $i$.

Proof of claim 2. (1) In a pure Nash equilibrium strategy profile for the discrete Voronoi game, if a player locates on a vertex $v_{j}^{i}(1 \leq j \leq t)$ and no player locates on $v_{j^{\prime}}^{i}$ for any $j^{\prime}>j$, then there exists another player located on the same vertex $v_{j}^{i}$ or on the adjacent vertex $v_{j-1}^{i}$.

(2) In a pure Nash equilibrium strategy profile for the information diffusion game, if a player locates on a vertex $v_{j}^{i}(1 \leq j \leq t)$ and no player locates on $v_{j^{\prime}}^{i}$ for any $j^{\prime}>j$, then there exists another player locating on the adjacent vertex $v_{j-1}^{i}$. 
By these claims, the only possible strategy profile for a candidate of a pure Nash equilibrium is $\left(v_{0}, v_{1}^{1}, v_{1}^{2}, \ldots, v_{1}^{k-1}\right)$. However, the player locating on $v_{0}$ can increase his/her utility by moving into $v_{2}^{i}$ for some $i$. Thus, this strategy profile is not a pure Nash equilibrium.

Theorem 18 For at least 3 players, there exist trees on which any pure Nash equilibria for neither the discrete Voronoi game nor the information diffusion game are admitted.

The characterization of the existence of pure Nash equilibria for both games in more general graph classes will be an interesting open problem for future works.

\section{Acknowledgements}

We would like to thank Satoshi Murai and Koji Nuida for helpful comments. This research is supported in part by the MEXT Grant-in-Aid for Scientific Research (C) No. 25350434.

\section{References}

[1] N. Alon, M. Feldman, A. D. Procaccia, and M. Tennenholtz, A note on competitive diffusion through social networks, Information Processing Letters, 110, 221-225 (2010).

[2] A. Borodin, Y. Filmus, and J. Oren, Threshold models for competitive influence in social networks, Internet and Network Economics, LNCS, 6484, 539-550 (2010)

[3] C. Dürr and N. K. Tang, Nash equilibria in Voronoi games on graphs, Proceedings of the 15th European Symposium on Algorithms, 17-28 (2007).

[4] S. Goyal and M. Kearns, Competitive contagion in networks, 44th ACM Symposium on Theory of Computing, 759-774 (2012).

[5] H. Hotelling, Stability in competition, The Economic Journal, 39, 41-57 (1929).

[6] A. Ito, and M. Muramatsu, A note on Nash equilibria in competitive diffusion games, Abstracts of the 2012 Spring National Conference of Operations Research Society of Japan, 212-213 (2012) in Japanese.

[7] M. Mavronicolas, B. Monien, V. G. Papadopoulou, and F. Schoppmann, Voronoi games on cycle graphs, Proceedings of the 33rd International Symposium on Mathematical Foundations of Computer Science (MFCS), 503-514 (2008).

[8] L. Small, and O. Mason, Nash equilibria for competitive information diffusion on trees, Information Processing Letters, 113, 217-219 (2013).

[9] R. Takehara, M. Hachimori, and M. Shigeno, A comment on pure-strategy Nash equilibria in competitive diffusion games, Information Processing Letters, 112, 59-60 (2012). 
[10] S. Teramoto, E. Demaine, and R. Uehara, Voronoi game on graphs and its complexity, Proceedings of the 2nd IEEE Symposium on Computational Intelligence and Games, 265-271 (2006).

[11] V. Tzoumas, C. Amanatidis, and E. Markakis, A game-theoretic analysis of a competitive diffusion process over social networks, Internet and Network Economics, LNCS, 7695, 1-14 (2012).

\section{Appendix: proof for Theorem 4}

Our proof for Theorem 4 is analogous to Mavronicolas et al. [7]. Particularly, we employ their arguments for the middle vertices of the paths. On the other hand, for the ends of the paths we need careful treatments. We provide here the full proof, though it includes similar discussions as [7]. As is remarked after Theorem 4, in this appendix we assume $2 n \geq k$.

We first prove that (V1)-(V6) are necessity conditions.

Lemma 19 If a strategy profile does not satisfy (V1), then there exists a player whose utility is improved by relocation.

Proof. Assume that $c_{j} \geq 3$, where $j \neq 1$ nor $\ell$. If $\theta_{j-1}=\theta_{j}-1$ and $\theta_{j+1}=\theta_{j}+1$ hold, then we have $\tilde{u}_{j}=\frac{1}{c_{j}} \leq \frac{1}{3}$, since $\left|L_{j-1}\right|=\left|L_{j}\right|=0$. By relocating a player on $\theta_{j}$ to a vertex that is chosen by at most one player, his/her utility increases to at least $\frac{1}{2}$. (Remark that such a vertex exists by the assumption $2 n \geq k$.) If either $\theta_{j-1}<\theta_{j}-1$ or $\theta_{j+1}>\theta_{j}+1$ holds, without loss of generality, we assume that $\left|L_{j-1}\right| \leq\left|L_{j}\right|$. Then, we have

$$
\tilde{u}_{j} \leq \frac{2\left\lfloor\frac{\left|L_{j}\right|}{2}\right\rfloor+1}{c_{j}}+2 \frac{\delta\left(\left|L_{j}\right|\right)}{c_{j}+1} \leq \frac{2\left\lfloor\frac{\left|L_{j}\right|}{2}\right\rfloor+1}{3}+\frac{\delta\left(\left|L_{j}\right|\right)}{2} .
$$

When a player on $\theta_{j}$ relocates to $\theta_{j}+1$, then his/her utility $\tilde{u}^{\prime}$ becomes

$$
\tilde{u}^{\prime}=\left\lfloor\frac{\left|L_{j}\right|-1}{2}\right\rfloor+1+\frac{1-\delta\left(\left|L_{j}\right|\right)}{1+c_{j+1}} .
$$

If $\delta\left(\left|L_{j}\right|\right)=0$ holds, then we obtain $\tilde{u}_{j} \leq \frac{\left|L_{j}\right|}{3}+\frac{1}{3}$ and $\tilde{u}^{\prime}>\frac{\left|L_{j}\right|}{2}$. It follows from $\left|L_{j}\right| \geq 2$ that $\tilde{u}_{j}<\tilde{u}^{\prime}$ holds. If $\delta\left(\left|L_{j}\right|\right)=1$ holds, then we obtain $\tilde{u}_{j} \leq \frac{\left|L_{j}\right|}{3}+\frac{1}{2}<\frac{\left|L_{j}\right|}{2}+\frac{1}{2}=\tilde{u}^{\prime}$. Therefor, a player on $\theta_{j}$ can improve his/her utility by moving onto $\theta_{j}+1$.

We next consider the case of $c_{1} \geq 3$. If $\ell=1$, then all players choose $\theta_{1}$ and obtain $\tilde{u}_{1} \leq \frac{n}{3}$. Since $\max \left\{\left|L_{0}\right|,\left|L_{1}\right|\right\} \geq \frac{n-1}{2}>\frac{n}{3}$ holds under $n \geq 4$, any player increases his/her utility by relocating to an appropriate neighbor vertex. Thus, we assume that $\ell>1$. It is easy to see as before that a player on $\theta_{1}$ can improve his/her utility if $\left|L_{0}\right|=\left|L_{1}\right|=0$. We assume at least one of $\left|L_{0}\right|>0$ or $\left|L_{1}\right|>0$ holds. In this case, we have

$$
\tilde{u}_{1} \leq \frac{\left|L_{0}\right|+1+\left\lfloor\frac{\left\lfloor L_{1} \mid\right.}{2}\right\rfloor}{3}+\frac{\delta\left(\left|L_{1}\right|\right)}{4} .
$$

If $\left|L_{0}\right| \leq\left\lfloor\frac{\left|L_{1}\right|}{2}\right\rfloor$ holds, then we obtain $\tilde{u}_{1} \leq \frac{2\left\lfloor\frac{\left|L_{1}\right|}{2}\right\rfloor+1}{3}+\frac{\delta\left(\left|L_{1}\right|\right)}{4} \leq \frac{\left|L_{1}\right|+1}{3}$. By relocating a player on $\theta_{1}$ to $\theta_{1}+1$, his/her utility increases to more than $\frac{\left|L_{1}\right|}{2}$, which is derived from 
(10). Thus, we have $\tilde{u}_{1}<\tilde{u}^{\prime}$. If $\left|L_{0}\right|>\left\lfloor\frac{\left|L_{1}\right|}{2}\right\rfloor$, that is equivalent to $\left|L_{0}\right| \geq \frac{\left|L_{1}\right|}{2}+\frac{1}{2}$, holds, we obtain $\tilde{u}_{1} \leq \frac{\left|L_{0}\right|+1+\frac{\left|L_{1}\right|-1}{2}}{3}+\frac{1}{4} \leq \frac{2\left|L_{0}\right|}{3}+\frac{1}{4}$. Thus, $\tilde{u}_{1}$ is less than $\left|L_{0}\right|$ which is the utility obtained by relocating a player on $\theta_{1}$ to $\theta_{1}-1$. Thus, in both cases, a player on $\theta_{1}$ can improve his/her utility by moving onto an appropriate neighbor vertex.

The case of $c_{\ell} \geq 3$ is also shown by the similar argument as the case of $c_{1} \geq 3$.

Lemma 20 If a strategy profile fulfills (V1) but does not satisfy (V2), then there exists a player whose utility is improved by relocation.

Proof. If $\left|L_{0}\right| \geq\lfloor\tilde{\gamma}\rfloor+1$ (resp. $\left|L_{\ell}\right| \geq\lfloor\tilde{\gamma}\rfloor+1$ ), then a player whose utility is $\tilde{\gamma}$ relocates to $\theta_{1}-1$ (resp. $\theta_{\ell}+1$ ) in order to increase his/her utility, where the new utility is given by $\left|L_{0}\right|\left(\operatorname{resp} .\left|L_{\ell}\right|\right)>\tilde{\gamma}$.

We next assume that $\left|L_{j}\right| \geq\lfloor 2 \tilde{\gamma}\rfloor$. If a player relocates to $\theta_{j+1}-1$, the his/her new utility $\tilde{u}^{\prime}$ becomes

$$
\tilde{u}^{\prime}=1+\left\lfloor\frac{\left|L_{j}\right|-1}{2}\right\rfloor+\frac{\delta\left(\left|L_{j}\right|-1\right)}{1+c_{j}} .
$$

If any one of (i) $\delta\left(\left|L_{j}\right|-1\right)=0$, (ii) $c_{j}=1$ and (iii) $\lfloor 2 \tilde{\gamma}\rfloor=2 \tilde{\gamma}$ is satisfied, we have $\tilde{u}^{\prime}>\tilde{\gamma}$. Thus, a player whose utility is $\tilde{\gamma}$ can increase his/her utility by moving onto $\theta_{j+1}-1$. So, we consider any $j$ with $\left|L_{j}\right| \geq\lfloor 2 \tilde{\gamma}\rfloor$ does not satisfy any of the cases (i), (ii) or (iii). For such a $j$, if $j \geq 2$ and $\left|L_{j}\right|>\left|L_{j-1}\right|$, then we have $\tilde{u}_{j}<\frac{\left|L_{j}\right|+1}{2}$. By relocating a player on $\theta_{j}$ to $\theta_{j+1}-1$, his/her utility becomes $\frac{\left|L_{j}\right|+1}{2}$. The case of $\left|L_{j}\right|<\left|L_{j-1}\right|$ also has a player whose utility can be improved, since $\left|L_{j-1}\right|>\lfloor 2 \tilde{\gamma}\rfloor$. Similarly, assume that $\left|L_{1}\right| \geq\lfloor 2 \tilde{\gamma}\rfloor$ and $\left|L_{1}\right|>2\left|L_{0}\right|$. Then, we have $\tilde{u}_{1}<\frac{\left|L_{1}\right|+1}{2}$ and the new utility after relocating to $\theta_{2}-1$ is $\frac{\left|L_{j}\right|+1}{2}$. When $\left|L_{1}\right|<2\left|L_{0}\right|$, we have $\left|L_{0}\right| \geq \frac{\lfloor 2 \tilde{\gamma}\rfloor+1}{2}>\lfloor\tilde{\gamma}\rfloor$, which we discussed in the first part of this proof. We can give a similar argument when $\left|L_{\ell-1}\right| \geq\lfloor 2 \tilde{\gamma}\rfloor$.

The remaining case is $\left|L_{j}\right|=p$ for all $j=1, \ldots, \ell-1,2\left|L_{0}\right|=2\left|L_{\ell}\right|=p$, where $p \geq\lfloor 2 \tilde{\gamma}\rfloor, \delta(p)=0$, and $c_{j}=2$ for all $j$. In this case, we have $\tilde{u}_{j}=\frac{p+1}{2} \geq \frac{\lfloor 2 \tilde{\gamma}\rfloor+1}{2}>\tilde{\gamma}$ for all $j$, which contradicts to the definition of $\tilde{\gamma}$.

Lemma 21 If a strategy profile fulfills (V2) but does not satisfy (V3), then there exists a player whose utility is improved by relocation.

Proof. Suppose that $c_{j}=1, c_{j+1} \geq 2$ and $\left|L_{j}\right|$ is odd. When $j=1$, the player on $\theta_{1}$ can increase his/her utility by relocating to $\theta_{1}+1$, since $\left|L_{1}\right| \geq 1$. When $j \neq 1$, we have

$$
\tilde{u}_{j} \leq \frac{\delta\left(\left|L_{j-1}\right|\right)}{1+c_{j-1}}+\left\lfloor\frac{\left|L_{j-1}\right|}{2}\right\rfloor+1+\frac{\left|L_{j}\right|-1}{2}+\frac{1}{3} .
$$

By relocating the player on $\theta_{j}$ to $\theta_{j}+1$, his/her utility $\tilde{u}^{\prime}$ becomes

$$
\tilde{u}^{\prime}=\frac{\delta\left(\left|L_{j-1}\right|+1\right)}{1+c_{j-1}}+\left\lfloor\frac{\left|L_{j-1}\right|+1}{2}\right\rfloor+1+\frac{\left|L_{j}\right|-1}{2} .
$$

If $\delta\left(\left|L_{j-1}\right|\right)=1$ holds, then we obtain $\tilde{u}_{j} \leq \frac{1}{1+c_{j-1}}+\frac{\left|L_{j-1}\right|+\left|L_{j}\right|}{2}+\frac{1}{3}<\frac{\left|L_{j-1}\right|+\left|L_{j}\right|}{2}+1=\tilde{u}^{\prime}$. If $\delta\left(\left|L_{j-1}\right|\right)=0$ holds, then we obtain $\tilde{u}_{j} \leq \frac{\left|L_{j-1}\right|+\left|L_{j}\right|}{2}+\frac{5}{6}$ and $\tilde{u}^{\prime}=\frac{1}{1+c_{j-1}}+\frac{\left|L_{j-1}\right|+\left|L_{j}\right|}{2}+\frac{1}{2}$. 
Thus, $\tilde{u}_{j}<\tilde{u}^{\prime}$ holds under $c_{j-1}=1$. If $c_{j-1} \geq 2$ and $j-1 \geq 2$, then the condition (i) of Lemma 5 implies that $\left|L_{j-1}\right|=\lfloor 2 \tilde{\gamma}\rfloor-1=\left|L_{j}\right|$. Thus, $\delta\left(\left|L_{j-1}\right|\right)=\delta\left(\left|L_{j}\right|\right)=1$, since $L_{j}$ is odd by assumption. The remaining case under $\delta\left(\left|L_{j-1}\right|\right)=0$ is $j=2$ and $c_{1} \geq 2$, that is, the case $c_{1} \geq 2, c_{2}=1$, and, $c_{3} \geq 2$. However, this is the condition of the exceptional case in (V3).

Finally, we check the statement for the case of $c_{1} \geq 2, c_{2}=1$ and $c_{3} \geq 2$ (The case of $c_{\ell} \geq 2, c_{\ell-1}=1$ and $c_{\ell-2} \geq 2$ is symmetric). If both of $\left|L_{1}\right|$ and $\left|L_{2}\right|$ are odd, then we obtain $\tilde{u}_{2} \leq \frac{2}{3}+\frac{\left|L_{j-1}\right|+\left|L_{j}\right|}{2}<\frac{\left|L_{j-1}\right|+\left|L_{j}\right|}{2}+1=\tilde{u}^{\prime}$, where $\tilde{u}^{\prime}$ is the utility obtained by the player on $\theta_{2}$ relocating to $\theta_{2}+1$.

Lemma 22 If a strategy profile fulfills (V3) but does not satisfy (V4), then there exists a player whose utility is improved by relocation.

Proof. Assume that $c_{j}=1,\left|L_{j-1}\right|=\left|L_{j}\right|=2 \tilde{\gamma}-1$ and $2 \tilde{\gamma}$ is even. From (V3), we have $c_{j-1}=c_{j+1}=1$. By relocating to $\theta_{j}$, a player whose utility is $\tilde{\gamma}$ increases his/her utility to at least

$$
\frac{1}{3}+\frac{\frac{\left|L_{j-1}\right|-1}{2}+1+\frac{\left|L_{j}\right|-1}{2}}{2}+\frac{1}{3}=\frac{2 \tilde{\gamma}-1}{2}+\frac{2}{3}>\tilde{\gamma}
$$

Lemma 23 If a strategy profile fulfills (V1) and (V3), but does not satisfy (V5), then there exists a player whose utility is improved by relocation.

Proof. Assume that $c_{j}=c_{j+1}=1$ and $\left|L_{j-1}\right|+\left|L_{j}\right|+1=\left|L_{j+1}\right|=2 \tilde{\gamma}-1$ hold whereas $2 \tilde{\gamma}$ is even. From (V3), we obtain $c_{j+2}=1$. If the player on $\theta_{j}$ relocates to $\theta_{j+1}$, then his/her utility becomes

$$
\frac{1}{2+c_{j-1}}+\frac{1}{2}\left(\frac{\left(\left|L_{j-1}\right|+\left|L_{j}\right|+1\right)-1}{2}+1+\frac{\left|L_{j+1}\right|-1}{2}\right)+\frac{1}{3} \geq \frac{1}{4}+\frac{1}{2}(2 \tilde{\gamma}-1)+\frac{1}{3}>\tilde{\gamma}
$$

while $\tilde{u}_{j} \leq \frac{\left|L_{j-1}\right|}{2}+1+\frac{\left|L_{j}\right|}{2} \leq \frac{2 \tilde{\gamma}-1}{2}+\frac{1}{2}=\tilde{\gamma}$. Hence, the player on $\theta_{j}$ can improve his/her utility.

The second implication can be shown in a similar way.

Lemma 24 If a strategy profile fulfills (V2) but does not satisfy (V6), then there exists a player whose utility is improved by relocation.

Proof. We only show the condition for $c_{1}=1$, as the the argument for the condition for $c_{\ell}=1$ is just symmetric.

If $\tilde{\gamma}<1$, then $\left|L_{j}\right|=0$ for all $0 \leq 0 \leq \ell$ and (V6) trivially holds. Hence we have $\tilde{\gamma} \geq 1$ in order that (V6) is not satisfied. It is obvious that the player on $\theta_{1}$ can increase his/her utility by relocating to $\theta_{1}+1$ if $\left|L_{1}\right| \geq 1$. Thus, we assume that $\left|L_{1}\right|=0$. We also assume that $\ell \geq 3$. Then, the condition (i) of Lemma 5 implies that $c_{2}=1$, since $\tilde{\gamma} \geq 1$ and $\lfloor 2 \tilde{\gamma}\rfloor-1 \geq 1$. When $\left|L_{0}\right| \leq \frac{\left|L_{2}\right|-1}{2}$, by relocating to $\theta_{2}$, the utility of the player on $\theta_{1}$ becomes

$$
\frac{\left(\left|L_{0}\right|+1\right)+1+\left\lfloor\frac{\left|L_{2}\right|}{2}\right\rfloor}{2}+\frac{\delta\left(\left|L_{2}\right|\right)}{2+c_{3}}>\frac{\left|L_{0}\right|+2+\frac{\left|L_{2}\right|-1}{2}}{2} \geq\left|L_{0}\right|+1
$$


which is greater than the original utility $\tilde{u}_{1}=\left|L_{0}\right|+1$. When $\left|L_{0}\right| \geq \frac{\left|L_{2}\right|}{2}+1$, by relocating $\theta_{1}$, the utility of the player on $\theta_{2}$ becomes

$$
\frac{\left|L_{0}\right|+1+\left\lfloor\frac{\left|L_{2}\right|+1}{2}\right\rfloor}{2}+\frac{\delta\left(\left|L_{2}\right|+1\right)}{2+c_{3}}>\frac{\left|L_{0}\right|+1+\frac{\left|L_{2}\right|}{2}}{2} \geq \frac{\left|L_{2}\right|}{2}+1,
$$

which is greater than the original utility $\tilde{u}_{2}=\left\lfloor\frac{\left|L_{2}\right|}{2}\right\rfloor+1+\frac{\delta\left(\left|L_{2}\right|\right)}{1+c_{3}} \leq \frac{\left|L_{2}\right|}{2}+1$.

To prove that (V1)-(V6) give a sufficient condition, we consider the following four cases of relocating a player in a strategy profile $(\boldsymbol{\theta}, \boldsymbol{c})$, as like Claim 1 in [7].

(C1) A player on $\theta_{j}$ with $c_{j}=1$ moves to a vertex in $L_{j-1} \cup L_{j}$.

(C2) A player on $\theta_{j}$ moves to a vertex in $L_{i}$ for the cases not covered by (C1).

(C3) A player on $\theta_{j}$ with $c_{j}=1$ moves to $\theta_{j-1}$ or $\theta_{j+1}$.

(C4) A player on $\theta_{j}$ moves to $\theta_{i}$ for the cases not covered by (C3).

Note that a strategy profile $(\boldsymbol{\theta}, \boldsymbol{c})$ is a pure Nash equilibrium if and only if neither case improves the utility of a player by relocation.

Lemma 25 The utility of the player is not improved by the relocation of (C1), if the strategy profile fulfills (V3) and (V6).

Proof. When $j=1$, the player can move to only $L_{0}$, since $\left|L_{1}\right|=0$ form (V6). It is obvious that his/her utility is not increased by moving into $L_{0}$. The case of $j=\ell$ is the same. When $j \neq 1, \ell$, without loss of generality, assume that the player moves to $\theta_{j}+a$ for $0<a \leq\left|L_{j}\right|$. The utility after moving is given by

$$
\tilde{u}^{\prime}=\frac{\delta\left(\left|L_{j-1}\right|+a\right)}{1+c_{j-1}}+\left\lfloor\frac{\left|L_{j-1}\right|+a}{2}\right\rfloor+1+\left\lfloor\frac{\left|L_{j}\right|-a}{2}\right\rfloor+\frac{\delta\left(\left|L_{j}\right|-a\right)}{1+c_{j+1}} .
$$

If $a$ is even, then we have $\tilde{u}_{j}=\tilde{u}^{\prime}$, since $\left\lfloor\frac{\left|L_{j-1}\right|-a}{2}\right\rfloor+\frac{\delta\left(\left|L_{j}\right|-a\right)}{1+c_{j+1}}=\left\lfloor\frac{\left|L_{j-1}\right|}{2}\right\rfloor+\frac{\delta\left(\left|L_{j}\right|\right)}{1+c_{j+1}}-\frac{a}{2}$. Otherwise, namely when $a$ is odd, then we have $\left\lfloor\frac{\left|L_{j}\right|-a}{2}\right\rfloor+\frac{\delta\left(\left|L_{j}\right|-a\right)}{1+c_{j+1}}>\left\lfloor\frac{\left|L_{j}\right|}{2}\right\rfloor+\frac{\delta\left(\left|L_{j}\right|\right)}{1+c_{j}+1}-\frac{a}{2}$ if and only if $c_{j+1} \geq 2$ and $\left|L_{j}\right|$ is odd. Almost such cases are forbidden by (V3). The remaining case is $c_{1}=2, c_{2}=1, c_{3}=2$ and either $\left|L_{1}\right|$ or $\left|L_{2}\right|$ is even (reps. $c_{\ell-2}=2$, $c_{\ell-1}=1, c_{\ell}=2$ and either $\left|L_{\ell-2}\right|$ or $\left|L_{\ell-1}\right|$ is even). For this case, we also have $\tilde{u}^{\prime}=\tilde{u}_{j}$ for any $a$.

Lemma 26 The utility of the player is not improved by the relocation of (C2), if the strategy profile fulfills (V2).

Proof. We denote by $\tilde{u}^{\prime}$ the utility of a player who moves to a vertex on $L_{i}$. If $i=0$ (reps. $i=\ell$ ), then $\tilde{u}^{\prime} \leq\left|L_{0}\right|\left(\operatorname{resp} .\left|L_{\ell}\right|\right) \leq\lfloor\tilde{\gamma}\rfloor \leq \tilde{\gamma}$. Otherwise, we have $\tilde{u}^{\prime} \leq \frac{\left|L_{i}\right|-1}{2}+1 \leq \frac{\lfloor 2 \tilde{\gamma}\rfloor}{2} \leq$ $\tilde{\gamma}$. Thus, the player can not increase his/her utility.

Lemma 27 The utility of the player is not improved by the relocation of (C3), if the strategy profile fulfills (V1), (V2), (V5) and (V6). 
Proof. Without loss of generality, we assume that the player on $\theta_{j}$ moves to $\theta_{j+1}$. The utility which the player obtains by moving is denoted by $\tilde{u}^{\prime}$.

We first consider the case of $j=1$. Since $\left|L_{0}\right|=\left\lfloor\frac{\left|L_{2}\right|}{2}\right\rfloor+\delta\left(\left|L_{2}\right|\right)$ from (V6), we obtain

$$
\tilde{u}^{\prime} \leq \frac{\left|L_{0}\right|+2+\left\lfloor\frac{\left|L_{2}\right|}{2}\right\rfloor}{2}+\frac{\delta\left(\left|L_{2}\right|\right)}{2+c_{3}} \leq\left|L_{0}\right|+1-\frac{\delta\left(\left|L_{2}\right|\right)}{6}
$$

while $\tilde{u}_{1}=\left|L_{0}\right|+1$

We next consider the case of $j \neq 1$ nor $\ell-1$. Since $\tilde{\gamma} \leq \tilde{u}_{j} \leq \frac{\left|L_{j-1}\right|+\left|L_{j}\right|}{2}+1$, we have $\left|L_{j-1}\right|+\left|L_{j}\right|+1 \geq 2 \tilde{\gamma}-1$. If $\left|L_{j-1}\right|+\left|L_{j}\right|+1>2 \tilde{\gamma}-1$ or $\left|L_{j+1}\right|<2 \tilde{\gamma}-1$, then $\left|L_{j-1}\right|+\left|L_{j}\right| \geq\left|L_{j+1}\right|$ holds. Thus, we have

$$
\begin{aligned}
\tilde{u}^{\prime} & \leq \frac{\delta\left(\left|L_{j-1}\right|+\left|L_{j}\right|+1\right)}{2+c_{j+1}}+\frac{\left\lfloor\frac{\left|L_{j-1}\right|+\left|L_{j}\right|+1}{2}\right\rfloor+1+\left\lfloor\frac{\left|L_{j+1}\right|}{2}\right\rfloor}{1+c_{j+1}}+\frac{\delta\left(\left|L_{j+1}\right|\right)}{2+c_{j+1}} \\
& \leq \frac{\delta\left(\left|L_{j-1}\right|+\left|L_{j}\right|+1\right)}{2+c_{j+1}}+\frac{\left\lfloor\frac{\left|L_{j-1}\right|+\left|L_{j}\right|+1}{2}\right\rfloor+1+\left\lfloor\frac{\left|L_{j+1}\right|+\left|L_{j}\right|}{2}\right\rfloor}{1+c_{j+1}}+\frac{\delta\left(\left|L_{j-1}\right|+\left|L_{j}\right|\right)}{2+c_{j+1}} \\
& =\frac{1}{2+c_{j+1}}+\frac{\left|L_{j-1}\right|+\left|L_{j}\right|+1}{1+c_{j+1}} \\
& \leq \frac{1}{3}+\frac{\left|L_{j-1}\right|+\left|L_{j}\right|}{2}+\frac{1}{1+c_{j+1}} \\
& \leq \tilde{u}_{j} .
\end{aligned}
$$

If $\left|L_{j-1}\right|+\left|L_{j}\right|+1=2 \tilde{\gamma}-1=\left|L_{j+1}\right|$, then we have $\tilde{u}^{\prime} \leq \frac{2 \delta(2 \tilde{\gamma}-1)}{2+c_{j+1}}+\frac{2\left\lfloor\frac{2 \tilde{\gamma}-1}{2}\right\rfloor+1}{1+c_{j+1}}$. When $c_{j+1}=2$, we have $\tilde{u}^{\prime} \leq \frac{2}{4}+\frac{2 \frac{2 \tilde{\gamma}-2}{2}+1}{3}<\tilde{\gamma} \leq \tilde{u}_{j}$. When $c_{j+1}=1$, then (V5) implies that $2 \tilde{\gamma}$ is odd. Thus, we obtain $\tilde{u}^{\prime} \leq \frac{2 \frac{2 \tilde{\gamma}-1}{2}+1}{2}=\tilde{\gamma} \leq \tilde{u}_{j}$.

Finally, we consider the case of $j=\ell-1$. This case also satisfies $\left|L_{j-1}\right|+\left|L_{j}\right|+1 \geq 2 \tilde{\gamma}-1$. Thus, from (V2), we have $\left|L_{\ell-2}\right|+\left|L_{\ell-1}\right|+1 \geq 2\left|L_{\ell}\right|-1$. Thus, it holds that

$$
\begin{aligned}
\tilde{u}^{\prime} & =\frac{\delta\left(\left|L_{\ell-2}\right|+\left|L_{\ell-1}\right|+1\right)}{c_{\ell-2}+1+c_{\ell}}+\frac{\left\lfloor\frac{\left|L_{\ell-2}\right|+\left|L_{\ell-1}\right|+1}{2}\right\rfloor+1+\left|L_{\ell}\right|}{1+c_{\ell}} \\
& \leq \frac{1}{3}+\frac{\left|L_{\ell-2}\right|+\left|L_{\ell-1}\right|+2}{1+c_{\ell}} .
\end{aligned}
$$

If $c_{\ell}=2$, then at least one of $\left|L_{\ell-2}\right|$ and $\left|L_{\ell-1}\right|$ is even due to (V3). Thus, we have

$$
\tilde{u}_{j}=\frac{\delta\left(\left|L_{\ell-2}\right|\right)}{1+c_{\ell-2}}+\left\lfloor\frac{\left|L_{\ell-2}\right|}{2}\right\rfloor+1+\left\lfloor\frac{\left|L_{\ell-1}\right|}{2}\right\rfloor+\frac{\delta\left(\left|L_{\ell-1}\right|\right)}{3} \geq \frac{1}{3}+\frac{\left|L_{\ell-2}\right|+\left|L_{\ell-1}\right|+1}{2} .
$$

Hence, we have $\tilde{u}^{\prime} \leq \tilde{u}_{j}$. If $c_{\ell}=1$, then derived from (V6) we have $\left|L_{\ell-1}\right|=0$ and $\left|L_{\ell}\right|=\left\lfloor\frac{\left|L_{\ell-2}\right|+1}{2}\right\rfloor$, which implies that

$$
\begin{aligned}
\tilde{u}^{\prime} & =\frac{\delta\left(\left|L_{\ell-2}\right|+1\right)}{2+c_{\ell-2}}+\frac{\left\lfloor\frac{\left\lfloor L_{\ell-2} \mid+1\right.}{2}\right\rfloor+1+\left\lfloor\frac{\left|L_{\ell-2}\right|+1}{2}\right\rfloor}{2} \\
& \leq \frac{\delta\left(\left|L_{\ell-2}\right|\right)}{1+c_{\ell-2}}+\left\lfloor\frac{\left|L_{\ell-2}\right|}{2}\right\rfloor+1=\tilde{u}_{j},
\end{aligned}
$$


where the last inequality comes from the fact that $\left|L_{\ell-2}\right|$ is even when $c_{\ell-2} \geq 2$ as required in (V3).

Lemma 28 The utility of a player will be not improved by the relocation of (C4), if the strategy profile fulfills (V2), (V4) and (V6).

Proof. We denote by $\tilde{u}^{\prime}$ the utility of a player who moves to a vertex on $\theta_{i}$.

Suppose that the player moves to $\theta_{1}$. If $c_{1}=1$, then the new utility $\tilde{u}^{\prime}$ becomes $\frac{\left|L_{0}\right|+1}{2} \leq \tilde{\gamma}$, since $\left|L_{1}\right|=0$. Otherwise, that is $c_{1} \geq 2$, we have

$$
\tilde{u}^{\prime} \leq \frac{1}{3}\left(\left|L_{0}\right|+1+\left\lfloor\frac{\left|L_{1}\right|}{2}\right\rfloor\right)+\frac{\delta\left(\left|L_{1}\right|\right)}{3+c_{2}} \leq \frac{1}{3}\left(\left|L_{0}\right|+1+\frac{\left|L_{1}\right|-1}{2}\right)+\frac{1}{4} \leq \frac{2}{3} \tilde{\gamma}+\frac{1}{4}<\tilde{\gamma} .
$$

The case that the player moves to $\theta_{\ell}$ is shown in a similar way.

We next suppose that the player moves to $\theta_{i}$ with $i \neq 1$, $\ell$. If $c_{i}=1$ and $\left|L_{i-1}\right|=$ $\left|L_{i}\right|=2 \tilde{\gamma}-1$, (V4) implies that $\left|L_{i-1}\right|$ and $\left|L_{i}\right|$ are even. Hence, we have

$$
\tilde{u}^{\prime} \leq \frac{\frac{\left|L_{i-1}\right|}{2}+1+\frac{\left|L_{i}\right|}{2}}{2}=\tilde{\gamma}
$$

If $\left|L_{i-1}\right|<2 \tilde{\gamma}-1$ or $\left|L_{i}\right|<2 \tilde{\gamma}-1$, we have

$$
\tilde{u}^{\prime} \leq \frac{1}{3}+\frac{\frac{\left|L_{i-1}\right|-1}{2}+1+\frac{\left|L_{i}\right|-1}{2}}{2}+\frac{1}{3} \leq \frac{4 \tilde{\gamma}-3}{4}+\frac{2}{3}<\tilde{\gamma}
$$

Finally, if $c_{i} \geq 2$, we have

$$
\tilde{u}^{\prime} \leq \frac{1}{4}+\frac{\frac{\left|L_{i-1}\right|-1}{2}+1+\frac{\left|L_{i}\right|-1}{2}}{3}+\frac{1}{4} \leq \frac{2 \tilde{\gamma}-1}{3}+\frac{1}{2}<\tilde{\gamma} .
$$

